\title{
Classical Sampling Theorems in the Context of Multirate and Polyphase Digital Filter Bank Structures
}

\author{
P. P. VAIDYANATHAN, SENIOR MEMBer, IEeE, AND VINCENT C. LIU, STUdent MEMBer, IEeE
}

\begin{abstract}
The sampling theorem has been generalized in several directions since its introduction during the first half of this century. Some of these include derivative sampling theorems and nonuniform sampling theorems. Both of these have also been reinterpreted in terms of a multichannel sampling framework. In the world of digital signal processing, multirate systems have gained substantial attention during the last $1 \frac{1}{2}$ decades. A system of this type is the maximally decimated analysis/synthesis filter bank, widely used in subband coding, voice privacy systems, and spectral analysis. Some of the generalizations of the sampling theorem can be understood by relating the sampling problem to the continuous-time version of the analysis/synthesis filter bank system, as was done by Papoulis and Brown. Basically, the recovery of a signal from "generalized samples" is a problem of designing appropriate linear filters called reconstruction (or synthesis) filters.

In this paper, this relation is first reviewed and explored further. New sampling theorems for the subsampling of sequences are derived by direct use of the digital filter bank framework. These results are related to the theory of perfect reconstruction in maximally decimated digital filter bank systems. One of these pertains to the subsampling of a sequence and its first few differences, and subsequent stable reconstruction at finite cost with no error. The reconstruction filters turn out to be multiplierless and of the FIR (finite impulse response) type. These ideas are extended to the case of two-dimensional signals, by use of a Kronecker formalism. This paper also considers the subsampling of band-limited sequences. A sequence $x(n)$ whose Fourier transform vanishes for $|\omega| \geq L \pi / M$, where $L$ and $M$ are integers with $L<M$, can in principle be represented by reducing the data rate by the amount $M / L$. Even though techniques are known to accomplish this reduction, this paper discusses a different method which consists in retaining only $L$ out of $M$ successive samples and discarding the rest (attractive when signal processing prior to transmission is prohibitive). The reconstruction problem in this context is addressed. The digital polyphase framework is used in this paper as a convenient tool for the derivation as well as mechanization of the sampling theorems.
\end{abstract}

\section{INTRODUCTION}

$\mathrm{T}$ HE sampling theorem, which was introduced several decades ago [1]-[3], has been extended by many authors into various generalized versions [4]-[6]. The simplest version of the theorem states that a continuous-time signal $x_{a}(t)$, band-limited to $|\Omega|<\sigma$, can be reconstructed from its equally spaced samples, if the sampling

Manuscript received October 13, 1987; revised March 5, 1988. This work was supported in part by the National Science Foundation under Grant DCI 8552579 , by the matching funds provided by Pacific Bell and General Electric Co., by Caltech's Programs in Advanced Technology grant sponsored by Aerojet General, General Motors, GTE, and TRW, and by the National Science Foundation under Grant MIP 8604456.

The authors are with the Department of Electrical Engineering, California Institute of Technology, Pasadena, CA 91125.

IEEE Log Number 8822365 . frequency is at least equal to the Nyquist frequency $\theta \triangleq$ $2 \sigma$. One of the earliest extensions of this theorem was stated by Shannon himself in his 1949 paper [1], which says that if $x_{a}(t)$ and its first $M-1$ derivatives are available, then uniformly spaced samples of these, taken at the reduced rate of $\Theta / M$, are sufficient to reconstruct $x_{a}(t)$. This result will be referred to as the derivative sampling theorem in this paper. A different type of extension called the nonuniform sampling theorem, which was stated by Black [7, p. 41] (and attributed to Cauchy, 1841!), has also been reviewed recently [4]-[6] in various contexts. Statements of this extension can be found in [4]-the essential message being that nonuniformly spaced samples of $x_{a}(t)$ can be used to reconstruct $x_{a}(t)$ to any desired accuracy, as long as the "average" sampling rate is at least equal to $\theta$. In accord with the traditionally used language [4], these will be collectively referred to as the "folk theorems." A very simple example of this type of result can be found in [40, pp. 557-558]. The use of "almost periodic functions" in the interpretation of sampling theorems is also worth noting in this context [28]. Some results on spectral representation of nonuniformly sampled waveforms can be found in [38].

An excellent tutorial review of the sampling theorem and several generalizations, both for one-dimensional and multidimensional signals, can be found in [4], along with an extensive bibliography from which proofs can be infered. The derivative sampling theorem and nonuniform sampling theorems were given a new and lucid interpretation in the fairly recent work by Papoulis [5], [39], by formulating a unified framework in terms of linear filtering operations prior to sampling. The Papoulis approach has a striking resemblance to the framework of filter banks, which is a discipline independently developed in the discrete-time domain during the last decade. The 1981 paper by Brown [6], which reinterprets the Papoulis framework, brings this resemblance closer, and also shows that the reconstruction of $x_{a}(t)$ from "generalized samples" is essentially a problem of designing a synthesis filter bank.

Recent advances in signal processing, which evolved in a manner independent of the above developments, have resulted in the discipline of multirate digital signal processing. A key feature here is the change of sampling rates at various stages of the system (hence the name multi- 
rate), in the form of decimation and interpolation of discrete-time sequences. Advantages and applications of such processing have been well understood [9]-[12], and will not be elaborated further. The purpose of this paper is to exploit further the close analogy between sampling-theorem frameworks and multirate filter banks, which involve the downsampling of sequences at various stages, followed by subsequent reconstruction.

Fig. 1 is a typical example of a multirate signal processing system, called the analysis/synthesis system. Here, the digital filters $H_{k}(z)$ are called analysis filters, and split the signal $x(n)$ into $M$ subbands in the frequency domain. The downgoing arrows in the figure represent the decimation operation by an integer factor of $M$. The $M$ decimated sequences are typically encoded and transmitted. At the receiver end, each of these decimated subband signals is "interpolated" (the upgoing arrows represent this; see the next section for formal definitions), and then recombined with the help of the synthesis filters $F_{k}(z)$ to form the reconstructed signal $\hat{x}(n)$. The main application of this system is in the subband coding of signals and images [10], [13]-[16]. The system also provides a model for short-time Fourier transforms as can be seen from the results of [17] (with the number of subbands not necessarily equal to the decimation ratio $M$ ). Our interest here, however, is in the mathematical framework offered by the system of Fig. 1. We shall see first that, if suitably adopted into the continuous time domain, it can be used for obtaining a simplified understanding of several versions of the sampling theorem. Moreover, a direct use of the discrete time structure of Fig. 1 gives rise to new downsampling theorems (or simply "sampling theorems") for sequences, some of which can be generalized to two (and higher)-dimensional sequences by further extensions of Fig. 1

Several interesting questions can be posed in connection with the reconstructibility of a sequence from various subsampled versions. These questions are closely analogous to the continuous time counterparts, but the answers often have certain qualitative differences. As an example, let us consider the derivative sampling theorem for a signal $x_{a}(t)$. In order to reconstruct exactly the value of $x_{a}(t)$ for a given $t$ from the samples of $x_{a}(t)$ and $\dot{x}_{a}(t)$, one has to perform an infinite amount of computation (because this involves ideal reconstruction filters and hence an infinite summation [6]). Let us look at the discrete-time version of this problem: let $x(n)$ be a sequence whose first difference is defined by $x_{1}(n)=x(n)-x(n-1)$. Suppose we decimate $x(n)$ and $x_{1}(n)$ by factors of 2 . Can we get back $x(n)$, for every $n$, from these decimated samples? Not surprisingly, the answer turns out to be yes, and moreover, exact reconstruction of $x(n)$ can be performed with a finite amount of computation (in fact, with only finite impulse response (FIR) filters). Reconstruction procedures are most easily obtained by treating this problem using the polyphase framework for multirate filter banks. Section III of this paper deals with this problem in greater detail, along with extensions to the two-dimensional case. Even though the difference operators used here are the

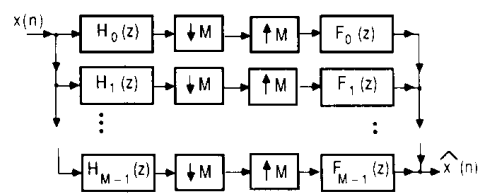

Fig. 1. The $M$-band multirate analysis/synthesis system.

(a)

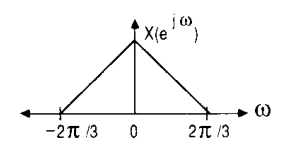

(b)

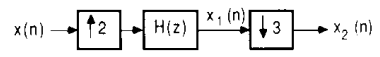

(c)

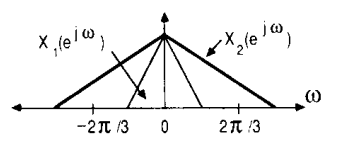

Fig. 2. A band-limited sequence, and a procedure to decimate it by the noninteger factor $3 / 2$.

same as those in [37], the purpose and problem statement in [37] are completely different.

As a second example, let $x(n)$ be a sequence band-limited to the region $|\omega|<2 \pi / 3$ [Fig. 2(a)]. We know that we can obtain a lower-rate signal with the same amount of information simply by creating a continuous time signal (by low-pass filtering) and resampling at two-thirds of the original rate, resulting in an obvious data-rate reduction. A direct discrete-time method for performing the same data-rate reduction is also well known [10] and is shown in Fig. 2(b). Here, the signal rate is first increased by a factor of 2 [by inserting a zero-valued sample between every two adjacent samples of $x(n)]$, and then filtered to ensure that the "image" created by the zero-insertion operation is eliminated. The resulting sequence $x_{1}(n)$, which is band-limited to $|\omega|<\pi / 3$, can be decimated by a factor of 3 without causing any aliasing. The output $x_{2}(n)$ has the same spectrum [Fig. 2(c)] as that of $x(n)$, except that it is stretched by the factor $3 / 2$. Now, is this the only way to perform data-rate reduction?

Imagine that the sequence $x(n)$ under consideration has been generated at a point where we do not have adequate signal processing facilities (such as computational and real-time resources). We wish to transmit the signal after performing the simplest possible data-rate reduction. It is then up to the receiver (who has sufficient funds, time, patience and other resources) to figure out the original $x(n)$. Such a scheme, for example, can be useful in mobile communications systems in which there are several moving transmitters, and a central fixed receiving station with sufficient signal processing facility. Now the wisdom gained from the "folk theorem" tells us that if we simply "drop" one out of every three samples of $x(n)$, thereby retaining the average "sampling rate" required by the 
signal bandwidth, we ought to be able to subsequently reconstruct $x(n)$. Not surprisingly, once again, this turns out to be true, even though the amount of computation for reconstruction required at the receiver-end increases with desired accuracy. In any case, if this problem is cast in the framework of polyphase digital filter banks, the solution looks surprisingly simple, leading to closed-form expressions for the synthesis filters. The mathematical tool for this purpose can be derived by adapting the results of [5]. Section IV gives a detailed treatment of this problem, along with expressions for the digital synthesis filters. Design examples, where such synthesis filters are approximated with linear phase FIR filters, are included.

Notations: The letters $\omega$ and $\Omega$ refer to the frequency variables for discrete and continuous signals, respectively. All signals under consideration are assumed to be of finite energy. A continuous time finite-energy signal $x_{a}(t)$ is said to be $\sigma$-band-limited (or $\sigma$-BL) if its Fourier transform exists, and is zero for $|\Omega| \geq \sigma$. The quantity $\Theta \triangleq 2 \sigma$ is the Nyquist rate. The letter $T$ denotes $2 \pi / \theta=$ $\pi / \sigma$, which is the Nyquist sampling period for the signal under consideration. A "band-limited sequence" $x(n)$ has a Fourier transform which vanishes for $\omega_{c} \leq|\omega| \leq$ $\pi$, where $\omega_{c}<\pi$. Theorems which deal with the subsampling of sequences are referred to as "sampling theorems for sequences."

For real-valued $\alpha, \operatorname{sgn}[\alpha]$ is equal to 1 for $\alpha>0$ and -1 for $\alpha<0$. Boldfaced quantities represent matrices and vectors. The row and column indexes of matrices and vectors are always assumed to begin at zero. Superscript $T$ and $\dagger$ represent transposition and transpose conjugation, respectively. A Vandermonde matrix [18] $\mathrm{V}$ is a square matrix ( say $M \times M$ ) whose lth column has the form [ 1 $\left.\begin{array}{llll}a_{l} & a_{l}^{2} & \cdots & a_{l}^{M-1}\end{array}\right]^{T}$. If $a_{l}$ are distinct (and none equal to zero) for $0 \leq l \leq M-1$, such a matrix is guaranteed to be nonsingular. Given an $I \times K$ matrix $\mathbf{A}$ and a $J \times L$ matrix $\mathbf{B}$, the Kronecker product [19], denoted by $\mathbf{A} \otimes$ $\mathbf{B}$, is defined to be the $I J \times K L$ matrix

$$
\mathbf{A} \otimes \mathbf{B}=\left[\begin{array}{cccc}
a_{0,0} \mathbf{B} & a_{0,1} \mathbf{B} & \cdots & a_{0, K-1} \mathbf{B} \\
a_{1,0} \mathbf{B} & a_{1,1} \mathbf{B} & \cdots & a_{1, K-1} \mathbf{B} \\
\vdots & \cdots & & \vdots \\
a_{I-1,0} \mathbf{B} & a_{I-1,1} \mathbf{B} & \cdots & a_{I-1, K-1} \mathbf{B}
\end{array}\right] .
$$

The following can be shown to hold for Kronecker products [19]:

$$
(A \otimes B)(C \otimes D)=(A C) \otimes(B D),
$$

provided the matrix products are all defined.

Purpose of the Paper, and Outline: The first purpose of the paper is to explicitly place in evidence the relation between digital filter banks and sampling theorems for continuous-time signals. The other purpose is to develop new sampling theorems for sequences (called differencesampling theorems) along with reconstruction procedures, both for 1-D and 2-D sequences. Finally, nonuniform sampling theorems for sequences are derived, along with reconstruction techniques.
In Section II we begin by reviewing certain topics in multirate digital signal processing, with emphasis on maximally decimated analysis/synthesis systems. These systems are closely related to the generalized sampling framework for continuous-time signals developed in [5] and [6]. In Section II we review this relation as well. Section III introduces the new family of difference sampling theorems for sequences. The procedure for reconstructing the original signal from the sampled subsequences is greatly facilitated by use of the polyphase framework [11], [12], which is defined and used in Section III. The difference sampling theorems are extended to the two-dimensional case in the same section. Section IV introduces nonuniform sampling theorems for sequences, by making use of the analysis/synthesis system introduced in Section II as a tool for representation of nonuniform sampling. As explained in Section IV, a simple proof for several nonuniform sampling theorems for continuous-time signals can be obtained from these results, along with reconstruction procedures using filter banks.

\section{Description of AnAlysis/Synthesis Systems}

The purpose of this section is to summarize some relevant results concerning the filter bank system of Fig. 1, and place in evidence its close connection with the generalized sampling theorems for continuous-time signals introduced earlier [5], [6]. The crucial building blocks in this structure are the decimator and the interpolator $[10]$, [12] which are separately shown in Fig. 3 . The $M$-fold decimator ( $M$ being an integer) is described by the inputoutput relation $y_{D}(n)=x(M n)$, which means that only the input samples occuring at times which are multiples of $M$ are retained. The "interpolator" is characterized by the input-output relation

$$
y_{l}(n)= \begin{cases}x(n / M) & \text { if } n \text { is a multiple of } M \\ 0 & \text { otherwise. }\end{cases}
$$

In other words, the interpolator inserts $M-1$ zero-valued samples between adjacent samples of $x(n)$. Even though this operation is not "interpolation" as understood intuitively, the name interpolator has become more or less standard and will be used in this paper. The operation of (3) can be described in the $z$ domain by $Y_{I}(z)=X\left(z^{M}\right)$, so that $Y_{I}\left(e^{j \omega}\right)=X\left(e^{j M \omega}\right)$. Thus, $Y_{I}\left(e^{j \omega}\right)$ has period $2 \pi / M$. Fig. 4 (b) shows this effect which is called imaging. The images, which occur in the region outside $-\pi / M$ $<\omega<\pi / M$, can be eliminated, if desired, by low-pass filtering.

The effect of the decimator in the transform domain is more involved. Since the time scale shrinks by a factor of $M$, this results in a transform-domain stretching. The expression for $Y_{D}\left(e^{j \omega}\right)$ is a sum of uniformly shifted versions [10] of $X\left(e^{j \omega / M}\right)$, viz., $X\left(e^{j(\omega-2 \pi m) / M}\right), 0 \leq m \leq$ $M-1$, i.e.,

$$
Y_{D}(z)=\frac{1}{M} \sum_{m=0}^{M-1} X\left(z^{1 / M} W^{m}\right)
$$




$$
\begin{aligned}
& x(n) \rightarrow \downarrow M \rightarrow y_{D}(n) \\
& \text { The } M \text {-fold decimator } \\
& x(n) \rightarrow f M \rightarrow y_{1}(n) \\
& \text { The } M \text {-fold interpolator }
\end{aligned}
$$

Fig. 3. The basic multirate building blocks.

(a)

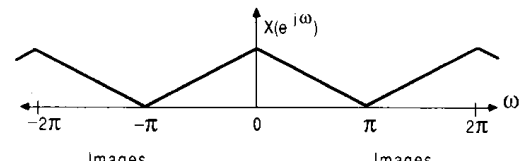

(b)

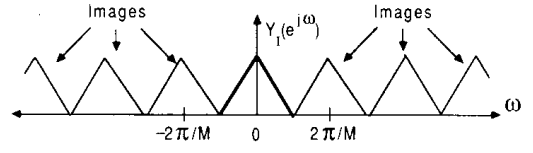

(c)

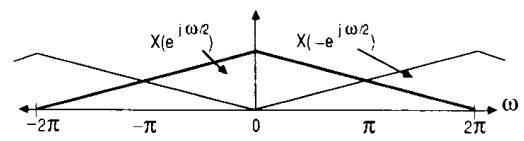

Fig. 4. Transform-domain effects of decimation and interpolation.

where $W=e^{-j 2 \pi / M}$. If the sequence $x(n)$ is not bandlimited to the region $|\omega|<\pi / M$, decimation causes aliasing. Under this condition, the terms with $m>0$ can have an overlap with the $m=0$ term, in the region $0 \leq$ $\omega \leq \pi$, as demonstrated in Fig. 4(c), for $M=2$. If an $\boldsymbol{M}$-fold interpolator follows an $\boldsymbol{M}$-fold decimator (as happens several times in Fig. 1), the result is stretching followed by compression in the frequency domain (Fig. 5). As long as $x(n)$ is band-limited to $|\omega|<\pi / M$, the overlaps in Fig. 5 are absent.

\section{A. Equations Governing the Analysis/Synthesis System}

With the above descriptions for decimators and interpolators, one can immediately write down the relation between $x(n)$ and the reconstructed signal $\hat{x}(n)$ in Fig. 1. This relation is [12], [20]-[24]

$$
\hat{X}(z)=\frac{1}{M} \sum_{m=0}^{M-1} X\left(z W^{m}\right) \sum_{k=0}^{M-1} H_{k}\left(z W^{m}\right) F_{k}(z),
$$

i.e., $\hat{X}(z)$ has the form $X(z) \sum_{k=0}^{M-1} H_{k}(z) F_{k}(z) / M$ plus terms containing $X(z W), X\left(z W^{2}\right)$, and so on. So $\hat{X}(z)$ is a linear combination of $X(z)$ and the alias-components $X\left(z W^{m}\right), m \neq 0$. The right-hand side of (5) can be expressed as $\mathbf{X}^{T}(z) \mathbf{H}(z) \mathbf{f}(z) / M$ where

$$
\begin{aligned}
\mathbf{X}^{T}(z) & =\left[\begin{array}{llll}
X(z) & X(z W) & \cdots & X\left(z W^{M-1}\right)
\end{array}\right], \\
\mathbf{f}^{T}(z) & =\left[\begin{array}{llll}
F_{0}(z) & F_{1}(z) & \cdots & F_{M-1}(z)
\end{array}\right]
\end{aligned}
$$

and

$$
[\mathbf{H}(z)]_{k m}=H_{m}\left(z W^{k}\right), \quad 0 \leq k, m \leq M-1 .
$$

For arbitrary $X(z)$, the alias components $X\left(z W^{m}\right), m>$ 0 , which are present in $\hat{X}(z)$ can be cancelled only by

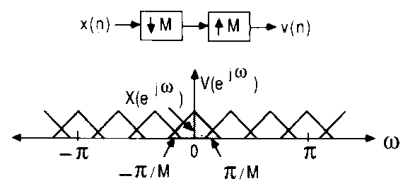

Fig. 5. The effect of decimation followed by interpolation.

choosing $H_{k}(z)$ and $F_{k}(z)$ such that

$$
\begin{gathered}
{\left[\begin{array}{cccc}
H_{0}(z) & H_{1}(z) & \cdots & H_{M-1}(z) \\
H_{0}(z W) & H_{1}(z W) & \cdots & H_{M-1}(z W) \\
\vdots & \vdots & & \vdots \\
H_{0}\left(z W^{M-1}\right) & H_{1}\left(z W^{M-1}\right) & \cdots & H_{M-1}\left(z W^{M-1}\right)
\end{array}\right]} \\
\cdot\left[\begin{array}{c}
F_{0}(z) \\
F_{1}(z) \\
\vdots \\
F_{M-1}(z)
\end{array}\right]=\left[\begin{array}{c}
M T(z) \\
0 \\
\vdots \\
0
\end{array}\right]
\end{gathered}
$$

where $T(z)$ is some function of $z$. The $M \times M$ matrix $\mathbf{H}(z)$, which was formulated in [21], is commonly called the alias-component matrix (AC-matrix for short). Given a set of $M$ analysis filters $H_{k}(z)$, if we can solve for the synthesis filters $F_{k}(z)$ such that $(8)$ holds for some $T(z)$, then the system of Fig. 1 is alias free. With aliasing thus cancelled, the system of Fig. 1 is time invariant and

$$
T(z) \triangleq \frac{1}{M} \sum_{k=0}^{M-1} H_{k}(z) F_{k}(z)
$$

describes the overall transfer function $\hat{X}(z) / X(z)$. If $H_{k}(z)$ and $F_{k}(z)$ are chosen such that $T(z)$ is equal to a delay, then $\hat{x}(n)=c x\left(n-n_{0}\right)$ and the system is said to have the perfect-reconstruction property [12], [24]-[26]. There exist systematic procedures for designing perfectreconstruction systems for arbitrary $M$, based on a class of transfer matrices called lossless matrices [12], [24], [26], [27], but these will not be considered further in this paper.

\section{B. The Generalized Sampling Framework for Continuous-Time Signals}

We shall now look into the relation between the discrete-time filter bank of Fig. 1, and the continuous-time analog shown in Fig. 6. This relation gives us additional insight, helping us to derive new sampling results, and redrive existing ones. The network of Fig. 6 was first used by Brown [6] to give a reinterpretation of the generalized sampling framework of Papoulis. In this system, $H_{a, k}(s)$ and $F_{a, k}(s)$ are analog filters, respectively, called analysis and synthesis filters. The input $x_{a}(t)$ is $\sigma$-band-limited, with Nyquist rate $\theta=2 \sigma$. The filtering operation is followed by the sampling of the continuous time signals at a rate $2 \sigma / M$, which is $M$ times smaller than the Nyquist rate $\theta$. In other words, the sampling period for each $x_{a, k}(t)$ is 


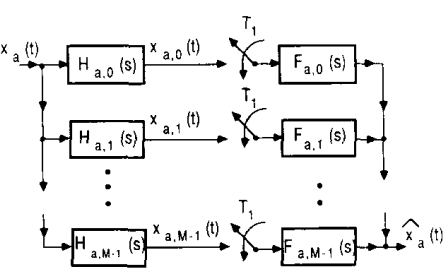

Fig. 6. The multichannel sampling framework for continuous-time signals.

$T_{1}=M T=M \pi / \sigma$ (with $T \triangleq 2 \pi / \theta=\pi / \sigma$ ). As the filters $H_{a, k}(s)$ are not ideal band-limiting functions, the undersampling of the filtered signals $X_{a, k}(s)$ results in aliasing. Since the sampled version of $x_{a, k}(t)$ has the transform

$$
\frac{1}{T_{1}} \sum_{m=-\infty}^{\infty} X_{a, k}\left(j \Omega-j \frac{2 \pi m}{T_{1}}\right)
$$

$\hat{X}_{a}(s)$ can be expressed in terms of $X_{a}(s), H_{a, k}(s)$, and $F_{a, k}(s)$ as

$$
\begin{aligned}
\hat{X}_{a}(s)= & \frac{1}{T_{1}} \sum_{m=-\infty}^{\infty} X_{a}\left(s-j \frac{2 \pi m}{T_{1}}\right) \\
& \cdot \sum_{k=0}^{M-1} H_{a, k}\left(s-j \frac{2 \pi m}{T_{1}}\right) F_{a, k}(s) .
\end{aligned}
$$

Once again, the terms in (11) corresponding to $m \neq 0$ are the aliasing terms. If these are somehow cancelled off by appropriate combinations of $H_{a, k}(s)$ and $F_{a, k}(s)$, the resulting system is time invariant with transfer function

$$
T_{a}(s)=\frac{1}{T_{1}} \sum_{k=0}^{M-1} H_{a, k}(s) F_{a, k}(s)
$$

If the transfer functions $H_{a, k}(s), F_{a, k}(s)$ can be chosen such that (12) equals a delay, then we have perfect reconstruction.

If we choose the analysis filters as $H_{a, k}(s)=s^{k}$, this corresponds to differentiation. It is possible in this case to show [6] that there will indeed exist synthesis filters $F_{a, k}(s)$ (not necessarily realizable) such that we can reconstruct $x_{a}(t)$ perfectly. This gives us a justification of the family of derivative sampling theorems. As a second example, if we take $H_{a, k}(s)$ to be pure delays, the result can be used to derive nonuniform-sampling theorems.

The main difference between the formulations of Fig. 1 and Fig. 6 lies in the summation over $m$, which is finite in (5) and infinite in (11). If we write the conditions for alias cancellation as in (8), we have an infinite number of equations (one for each $m$ ), with each equation having $M$ terms (one for each $k$ ). The band-limited nature of $x_{a}(t)$, however, helps to reduce (11) into a finite summation, with only $M$ values of $m$ (even though the exact values of $m$ participating in the summation depend on the value of $\Omega$ under consideration, as we shall see). These principles are best demonstrated with an example, which follows next.
Example 2.1: Consider the case of two channels in Fig. 6 so that $M=2$. Perfect reconstruction [i.e., $\hat{X}_{a}(s)=$ $X_{a}(s)$ ] can be obtained [for arbitrary $\sigma$-band-limited $\left.x_{a}(t)\right]$ if the following equations are satisfied for all $m$ :

$$
\begin{aligned}
& H_{a, 0}\left(s-j \frac{2 \pi m}{T_{1}}\right) F_{a, 0}(s) \\
& \quad+H_{a, 1}\left(s-j \frac{2 \pi m}{T_{1}}\right) F_{a, 1}(s)=T_{1} \delta(m)
\end{aligned}
$$

where $\delta(m)$ is the unit pulse function (zero for $m \neq 0$ and unity for $m=0$ ). In this example, we have $T_{1}=2 T$ $=2 \pi / \sigma$ so that $2 \pi / T_{1}=\sigma$. Fig. 7 shows a possible sketch of $X_{a}(j \Omega-j \sigma m)$ for various values of $m$. If we choose the synthesis filters such that $F_{a, k}(j \Omega)$ is zero for $|\Omega| \geq \sigma$, the only values of $m$ that remain to be considered are $m=0,1,-1$. For $-\sigma<\Omega \leq 0$, only the terms in (13) corresponding to $m=0$ and $m=-1$ need to be considered. For $0 \leq \Omega<\sigma$, one has to consider only $m$ $=0,1$. In order to obtain perfect recovery of the signal, it therefore remains only to solve for $F_{a, k}(s)$ from the equations

$$
\left[\begin{array}{ll}
H_{a, 0}(j \Omega) & H_{a, 1}(j \Omega) \\
H_{a, 0}(j \Omega+j \sigma) & H_{a, 1}(j \Omega+j \sigma)
\end{array}\right]\left[\begin{array}{l}
F_{a, 0}(j \Omega) \\
F_{a, 1}(j \Omega)
\end{array}\right]=\left[\begin{array}{l}
T_{1} \\
0
\end{array}\right]
$$

for $-\sigma<\Omega \leq 0$ and

$$
\left[\begin{array}{ll}
H_{a, 0}(j \Omega) & H_{a, 1}(j \Omega) \\
H_{a, 0}(j \Omega-j \sigma) & H_{a, 1}(j \Omega-j \sigma)
\end{array}\right]\left[\begin{array}{l}
F_{a, 0}(j \Omega) \\
F_{a, 1}(j \Omega)
\end{array}\right]=\left[\begin{array}{l}
T_{1} \\
0
\end{array}\right]
$$

for $0 \leq \Omega<\sigma$. Assuming that the $2 \times 2$ matrices above are nonsingular in the indicated frequency ranges, it is possible to solve for $F_{a, 0}(j \Omega)$ and $F_{a, 1}(j \Omega)$ in each frequency band. For example, consider the case where $H_{a, 0}(s)=1$ and $H_{a, 1}(s)=s$. This corresponds to the situation where the signal $x_{a}(t)$ and its derivative are sampled at one-half the Nyquist rate. Solving for the synthesis filters $F_{a, k}(j \Omega)$, we obtain [6] $F_{a, 0}(j \Omega)=T_{1}(\sigma-$ $|\Omega|) / \sigma$, and $F_{a, 1}(j \Omega)=-j T_{1} \operatorname{sgn}[\Omega] / \sigma$ for $|\Omega| \leq \sigma$. With $F_{a, 0}(j \Omega)=F_{a, 1}(j \Omega)=0$ for $|\Omega|>\sigma$, the synthesis filters are completely defined. The corresponding impulse responses are $f_{a, 0}(t)=4 \sin ^{2}(\sigma t / 2) / \sigma^{2} t^{2}$, and $f_{a, 1}(t)=4 \sin ^{2}(\sigma t / 2) / \sigma^{2} t$. These filters are indeed unrealizable $\left(F_{a, 0}(s)\right.$ has poles at $s=\infty$ and $F_{a, 1}(s)$ is an ideal Hilbert transformer). This is not surprising; indeed, the simplest version of sampling theorem (Nyquist's theorem) also requires an ideal low-pass filter for theoretically perfect reconstruction.

For arbitrary $M$, the situation is similar. Assuming that $F_{a, k}(j \Omega)=0$ for $|\Omega| \geq \sigma$, it only remains to cancel aliasing in the region $|\Omega|<\sigma$. Once again, because of the $\sigma$-band-limited nature of $x_{a}(t)$, it can be verified that for a given $\Omega$ in this region, only $M$ values of $m$ have nonzero contributions. As pointed out in [5], if the anal- 


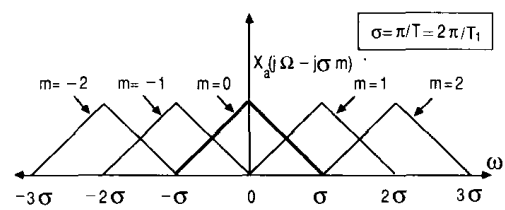

Fig. 7. A band-limited spectrum, and the alias components for various values of $m$. Here $M=2$.

ysis filters are chosen to be $H_{a, k}(s)=s^{k}$, the $M \times M$ matrices involved are Vandermonde matrices with distinct columns, and therefore nonsingular for all $\Omega$. This guarantees existence of synthesis filters (not necessarily realizable) for perfect recovery of $x_{a}(t)$.

\section{Sampling Theorems for Sequences Based on FINITE DIFFERENCES}

Consider the discrete-time structure of Fig. 1, where $H_{k}(z)$ and $F_{k}(z)$ are digital filters. Unlike the continuous time case, it is possible to find several realizable examples of $H_{k}(z)$ and $F_{k}(z)$, which give rise to perfect reconstruction [i.e., $\hat{x}(n)=c x\left(n-n_{0}\right)$ ]. Such examples can be found in [12] and [24]-[26]. In principle, these can be used to generate new sampling theorems for sequences, and there exist an infinite number of these! The purpose of this section is to consider a particular subclass of such systems, where $H_{k}(z)$ are discrete difference operators. In this case, it is possible to find surprisingly simple closed-form expressions for the synthesis filters $F_{k}(z)$ which, in addition, are FIR.

\section{A. Theorems for One-Dimensional Sequences}

It is best to begin with an example. Consider the case $M=2$, with analysis filters

$$
H_{0}(z)=1, \quad H_{1}(z)=1-z^{-1} .
$$

Let $x_{0}(n)$ and $x_{1}(n)$ be the outputs of $H_{0}(z)$ and $H_{1}(z)$, respectively. Then $x_{0}(n)=x(n)$ and $x_{1}(n)=x(n)-$ $x(n-1)$, i.e., $x_{1}(n)$ is the first difference of the sequence $x(n)$. After decimating $x_{0}(n)$ and $x_{1}(n)$ by factors of 2 , how do we get back the sequence $x(n)$ ? For this, we employ (8): we have to find $F_{0}(z)$ and $F_{1}(z)$ such that (8) holds with $T(z)$ equal to a delay. The relevant equations to be solved are

$$
\left[\begin{array}{ll}
1 & 1-z^{-1} \\
1 & 1+z^{-1}
\end{array}\right]\left[\begin{array}{l}
F_{0}(z) \\
F_{1}(z)
\end{array}\right]=\left[\begin{array}{l}
2 T(z) \\
0
\end{array}\right],
$$

since $W=e^{-j 2 \pi / M}=-1$ in this case. Solving for $F_{0}(z)$ and $F_{1}(z)$, we obtain $F_{0}(z)=1+z^{-1}, F_{1}(z)=-1$, along with $T(z)=z^{-1}$, so that $\hat{x}(n)=x(n-1)$. As a motivation for this kind of undersampling, consider a hypothetical sequence $x(n)$ as in Fig. 8. Assume that the sample values $x(n)$ are 16-bit, nonnegative, fixed-point numbers such that the difference $x(n)-x(n-1)$ has magnitude not exceeding 256 . The difference can therefore be represented by 8 rather than 16 bits, and a sign

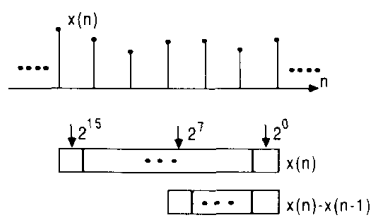

Fig. 8. The binary register patterns for a hypothetical sequence and its first difference.

bit. Clearly, a decimated version of $x(n)$ along with a decimated version of $x(n)-x(n-1)$ can be transmitted with fewer bits per unit time than $x(n)$ itself. The simple reconstruction filters $F_{0}(z), F_{1}(z)$ above enable us to recover $x(n)$ subsequently without error. In practice, the properties of the first difference are typically more complicated than this, but any strong correlation between successive samples can be exploited in this manner. Such correlation is, of course, the well-known motivation for many data-compression techniques known today [31].

For arbitrary $M$, if the analysis filters in Fig. 1 have the form

$$
H_{k}(z)=\left(1-z^{-1}\right)^{k}, \quad 0 \leq k \leq M-1,
$$

we can once again invoke (8) to solve for the synthesis filters. The AC-matrix $\mathbf{H}(z)$ has $(m, k)$ element of the form $\left(1-z^{-1} W^{-m}\right)^{k}$ and, hence, $\mathbf{H}^{T}(z)$ is a Vandermonde matrix. Since its determinant [18] is a product of factors of the form $\left(1-z^{-1} W^{-l}-1+z^{-1} W^{-m}\right)$ with $m \neq l$, this determinant is a constant times a delay, and is nonsingular on the unit circle. Accordingly, by choosing $T(z)$ to be an appropriate delay, we can uniquely solve for $F_{k}(z)$ which are guaranteed to be FIR filters.

The Polyphase-Component Framework: It is of interest to derive closed-form expressions for these synthesis filters. It is known that the use of the polyphase formulation [11], [12], [24] leads to substantial simplification during such derivations. We shall therefore define and use the polyphase approach next.

Let $H(z)=\Sigma_{n=-\infty}^{\infty} h(n) z^{-n}$ be any transfer function. Let us decompose the impulse response sequence $h(n)$ into $M$ subsequences as follows: $e_{m}(n)=h(m+n M)$, $0 \leq m \leq M-1$. In other words, $e_{m}(n)$ is an $M$-fold decimated version of $h(m+n)$. Let $E_{m}(z)$ denote the $z$ transform of $e_{m}(n)$. We then have $H(z)=$ $\Sigma_{m=0}^{M-1} z^{-m} E_{m}\left(z^{M}\right)$. This is called the polyphase representation of $H(z)$, and $E_{m}(z)$ are the polyphase components of $H(z)$ [see Fig. 9(a)]. This representation has been found to be very useful in the design of uniform filter banks [10], [11], [23], and in the implementation of "decimation filters" [10]. An alternative polyphase representation [Fig. 9(b)] takes the form $H(z)=$ $\sum_{m=0}^{M-1} z^{-(M-1-m)} R_{m}\left(z^{M}\right)$. This is obtained merely by defining $R_{m}(z)=E_{M-1-m}(z)$, and is found to be convenient in designing synthesis banks and interpolation filters [10].

Let us express each analysis filter in Fig. 1 in the polyphase form 


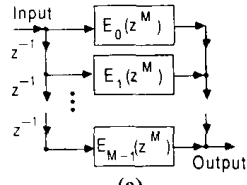

(a)

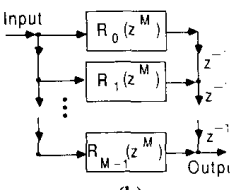

(b)

Fig. 9. Two types of polyphase representations of a discrete-time transfer function.

$$
H_{k}(z)=\sum_{m=0}^{M-1} z^{-m} E_{k m}\left(z^{M}\right)
$$

where $E_{k m}(z)$ are the polyphase components of $H_{k}(z)$. Similarly, let

$$
F_{k}(z)=\sum_{m=0}^{M-1} z^{-(M-1-m)} R_{m k}\left(z^{M}\right) .
$$

This enables us to represent the analysis/synthesis system as shown in Fig. 10, where $\mathbf{E}(z)=\left[E_{k m}(z)\right]$ and $\mathbf{R}(z)$ $=\left[R_{m k}(z)\right]$ are $M \times M$ transfer matrices. This representation has been used in the past to obtain necessary and sufficient conditions for perfect reconstruction [24]. In this section, we use this representation to find out expressions for the synthesis filters $F_{k}(z)$ which should be used in conjunction with (18) so as to obtain $\hat{x}(n)=c x\left(n-n_{0}\right)$.

If the matrix $\mathbf{R}(z)$ is equal to $\mathbf{E}^{-1}(z)$, then the structure of Fig. 10 reduces to Fig. 11, for which we can verify easily [24] that $\hat{x}(n)=x(n-M+1)$. For the choice of analysis filters as in (18), the $M \times M$ matrix $\mathbf{E}(z)$ becomes

$$
\mathbf{E}=\left[\begin{array}{rrrrrr}
1 & 0 & 0 & 0 & \cdots & 0 \\
1 & -1 & 0 & 0 & \cdots & 0 \\
1 & -2 & 1 & 0 & \cdots & 0 \\
1 & -3 & 3 & -1 & \cdots & 0 \\
\vdots & & & & \cdots & \vdots
\end{array}\right]
$$

In words, the rows of $\mathbf{E}(z)$ are constants, equal to the binomial coefficients occurring in $\left(1-z^{-1}\right)^{k}$. In the discussions to follow, $\mathbf{E}$ in (21) will be called a binomial matrix. Clearly, it is a nonsingular matrix (all eigenvalues equal to \pm 1 ), and we can find a unique $\mathbf{R}=\mathbf{E}^{-1}$. The inverse turns out to be particularly simple, as stated below.

Lemma 3.1: The $M \times M$ binomial matrix $\mathbf{E}$ in (21) is its own inverse.

As a result, if we set $\mathbf{R}=\mathbf{E}$ in Fig. 10, we have a perfect reconstruction system! For example, with $M=3$, the synthesis filters can be obtained as

$$
\left[\begin{array}{lll}
F_{0}(z) & F_{1}(z) & F_{2}(z)
\end{array}\right]=\left[\begin{array}{lll}
z^{-2} & z^{-1} & 1
\end{array}\right]\left[\begin{array}{rrr}
1 & 0 & 0 \\
1 & -1 & 0 \\
1 & -2 & 1
\end{array}\right]
$$

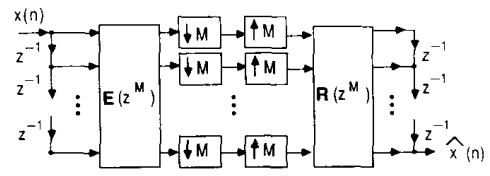

Fig. 10. Representation of analysis and synthesis banks in terms of polyphase components.

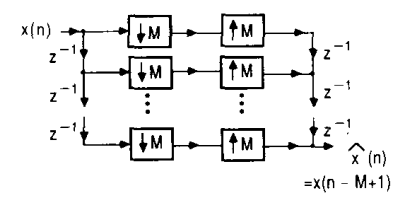

Fig. 11. A simple perfect-reconstruction system.

so that $F_{0}(z)=1+z^{-1}+z^{-2}, F_{1}(z)=-z^{-1}-2$, and $F_{2}(z)=1$. As a general rule, it can be verified that, with the analysis filters as in (18), the synthesis filter $F_{k}(z)$ computed with $\mathbf{R}=\mathbf{E}$ has order $M-1-k$ for $0 \leq k$ $\leq M-1$.

It now remains to prove Lemma 3.1. A proof based on combinatorial arguments can indeed be given, but we consider the following argument to be simpler: define

$$
\mathbf{e}\left(z^{-1}\right) \triangleq\left[\begin{array}{lllll}
1 & z^{-1} & z^{-2} & \cdots & z^{-(M-1)}
\end{array}\right]^{T} .
$$

It is then clear by definition by $\mathbf{E}$ that

$$
\mathbf{E e}\left(z^{-1}\right)=\mathbf{e}\left(1-z^{-1}\right)
$$

Thus, the effect of the operator $\mathbf{E}$ on $\mathbf{e}(x)$ is to replace the argument $x$ with $1-x$. As a result,

$$
\mathbf{E}^{2} \mathbf{e}\left(z^{-1}\right)=\mathbf{E e}\left(1-z^{-1}\right)=\mathbf{e}\left(z^{-1}\right) .
$$

Since $\mathbf{E}^{2} \mathbf{e}\left(z^{-1}\right)=\mathbf{e}\left(z^{-1}\right)$ holds for any $z$, we have

$$
\begin{aligned}
& \mathbf{E}^{2}\left[\begin{array}{llll}
\mathbf{e}\left(z_{0}^{-1}\right) & \mathbf{e}\left(z_{1}^{-1}\right) & \cdots & \mathbf{e}\left(z_{M-1}^{-1}\right)
\end{array}\right] \\
& \quad=\left[\begin{array}{llll}
\mathbf{e}\left(z_{0}^{-1}\right) & \mathbf{e}\left(z_{1}^{-1}\right) & \cdots & \mathbf{e}\left(z_{M-1}^{-1}\right)
\end{array}\right]
\end{aligned}
$$

for $M$ distinct values $z_{k}$ of $z$. The $M \times M$ matrix of e $\left(z_{k}^{-1}\right)$ 's in (26) is Vandermonde with distinct columns, and is therefore nonsingular. As a result, it follows that $\mathbf{E}^{2}=\mathbf{I}$, proving the lemma. The results of the above discussions can be summarized as follows.

Theorem 3.1-The Difference-Sampling Theorem: Let $x(n)$ be any sequence and let $x_{k}(n)$ be the $k$ th difference sequence, defined to be such that $X_{k}(z)=(1-$ $\left.z^{-1}\right)^{k} X(z)$. Then $x(n)$ can be recovered from $M$-fold decimated versions of $x_{k}(n), 0 \leq k \leq M-1$. The reconstruction filters $F_{k}(z), 0 \leq k \leq M-1$ are FIR with orders $M-1-k$, and are given by

$$
\begin{aligned}
& {\left[\begin{array}{llll}
F_{0}(z) & F_{1}(z) & \cdots & F_{M-1}(z)
\end{array}\right]} \\
& \quad=\left[\begin{array}{llll}
z^{-(M-1)} & z^{-(M-2)} & \cdots & 1
\end{array}\right] \mathbf{E} .
\end{aligned}
$$

The filters $\left(1-z^{-1}\right)^{k}$ are all high-pass filters. For reasons somewhat analogous to the continuous time differentiators, these filters tend to amplify computational noise for large $k$. However, since the coefficients in both $\mathbf{R}$ and 
$\mathbf{E}$ are integers, it is possible to perform all operations using integer (modulo-) arithmetic, thereby preventing roundoff errors altogether.

Other variations of the analysis filters, such as $H_{k}(z)$ $=\left(1-z^{-1}\right)^{i}\left(1+z^{-1}\right)^{k-i}$ which can provide low-pass, bandpass, and high-pass functions for appropriate choice of $i$ (with $0 \leq i \leq k$ ), are possible. With analysis filters of this form, we still have a lower triangular $\mathbf{E}$ matrix, with diagonal entries equal to \pm 1 . A unique $\mathbf{R}=\mathbf{E}^{-1}$ therefore exists, from which the reconstruction filters $F_{k}(z)$ can be found. These obviously remain FIR filters (with integer coefficients) of orders not exceeding $M-1$.

\section{$B$. Extension to 2-D Sequences}

Consider a two-dimensional (2-D) sequence $x\left(n_{1}, n_{2}\right)$ with transform $X\left(z_{1}, z_{2}\right)$. In this section we would like to extend Theorem 3.1 to the 2-D case by appropriately reformulating the filter bank of Fig. 1, and redefining difference sequences for the 2-D case. In the 2-D case, the analysis/synthesis system takes a more complicated form [16], [29], [30] with $M N$ ( rather than $M$ ) branches. The $(m, n)$ th branch is shown in Fig. 12, where $H_{m n}\left(z_{1}, z_{2}\right)$ and $F_{m n}\left(z_{1}, z_{2}\right)$ are analysis and synthesis filters, respectively. The downgoing arrow represents decimation by the factor $M$ in the horizontal direction and by the factor $N$ in the vertical direction. The upgoing arrows represent the insertion of $M-1$ zero-valued pixels between adjacent pixels in the horizontal direction, followed by the insertion of $N-1$ zero-valued pixels between adjacent pixels in the vertical direction. The signals $p_{m n}\left(n_{1}, n_{2}\right)$ are merely added to obtain the reconstructed signal $\hat{x}\left(n_{1}, n_{2}\right)$.

In the one-dimensional case, the simple filter bank shown in Fig. 11 has the perfect reconstruction property, and this fact was used in Section III-A to obtain Theorem 3.1. Our first task now will be to find the equivalent of Fig. 11 in the 2-D case. Essentially, we would like the set the analysis filters to be all possible combinations of the delays $z_{1}^{-m}$ and $z_{2}^{-n}$. To be more specific, if we let $H_{m n}\left(z_{1}, z_{2}\right)=z_{1}^{-m} z_{2}^{-m}$ for $0 \leq m \leq M-1,0 \leq n \leq N$ -1 , then the synthesis filters can be easily chosen to ensure perfect reconstruction. The following result has been shown in [16].

Lemma 3.2: In the filter bank of Fig. 12, if we let $H_{m n}\left(z_{1}, z_{2}\right)=z_{1}^{-m} z_{2}^{-n}$, then the choice of synthesis filters according to $F_{m n}\left(z_{1}, z_{2}\right)=z_{1}^{-(M-1-m)} z_{2}^{-(N-1-n)}$ results in perfect reconstruction, and we have $\hat{x}\left(n_{1}, n_{2}\right)=x\left(n_{1}\right.$ $\left.-M+1, n_{2}-N+1\right)$.

Consider a simple example with $M=N=2$. Let $H_{00}\left(z_{1}, z_{2}\right)=1, H_{10}\left(z_{1}, z_{2}\right)=1-z_{1}^{-1}, H_{01}\left(z_{1}, z_{2}\right)=1$ $-z_{2}^{-1}, H_{11}\left(z_{1}, z_{2}\right)=\left(1-z_{1}^{-1}\right)\left(1-z_{2}^{-1}\right)$ so that

$$
\left[\begin{array}{l}
H_{00}\left(z_{1}, z_{2}\right) \\
H_{10}\left(z_{1}, z_{2}\right) \\
H_{01}\left(z_{1}, z_{2}\right) \\
H_{11}\left(z_{1}, z_{2}\right)
\end{array}\right]=\left[\begin{array}{rrrr}
1 & 0 & 0 & 0 \\
1 & -1 & 0 & 0 \\
1 & 0 & -1 & 0 \\
1 & -1 & -1 & 1
\end{array}\right]\left[\begin{array}{l}
1 \\
z_{1}^{-1} \\
z_{2}^{-1} \\
z_{1}^{-1} z_{2}^{-1}
\end{array}\right] \text {. }
$$

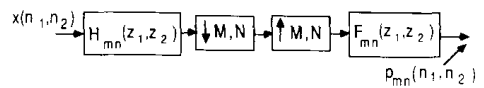

Fig. 12. The $(m, n)$ th branch of the two-dimensional analysis/synthesis system.

We can redraw the system as in Fig. 13, with the matrix A representing the $4 \times 4$ matrix in (28). If the matrix $\mathbf{B}$ in the figure can now be chosen to be $\mathbf{A}^{-1}$, then the resulting system has the perfect reconstruction property according to Lemma 3.2. It can be verified in this example that $\mathbf{A}^{-1}=\mathbf{A}$ so that the synthesis filters for perfect reconstruction become $F_{00}\left(z_{1}, z_{2}\right)=1+z_{1}^{-1}+z_{2}^{-1}+$ $z_{1}^{-1} z_{2}^{-1}, F_{10}\left(z_{1}, z_{2}\right)=-1-z_{2}^{-1}, F_{01}\left(z_{1}, z_{2}\right)=-1-$ $z_{1}^{-1}$, and $F_{11}\left(z_{1}, z_{2}\right)=1$. These are multiplierless FIR filters.

General Definition of Higher Order Differences: How do we generalize this result to the case of arbitrary $M$ and $N$ ? The first step is to define 2-D differences appropriately; the second step is to identify the $\mathbf{A}$ matrix, and invert it. Let us begin by reconsidering analysis and synthesis filters of the form $z_{1}^{-m^{m}} z_{2}^{-n}$, and $z_{1}^{-(M-1-m)} z_{2}^{-(N-1-n)}$, respectively. Let $\mathbf{e}\left(z_{1}, z_{2}\right)$ denote the $M N \times 1$ vector of these analysis filters, i.e.,

$$
\begin{aligned}
{\left[\mathbf{e}\left(z_{1}, z_{2}\right)\right]_{m+M n}=z_{1}^{-m} z_{2}^{-n}, } & 0 \leq m \leq M-1, \\
& 0 \leq n \leq N-1,
\end{aligned}
$$

and let $\mathbf{r}\left(z_{1}, z_{2}\right)$ be the $M N \times 1$ vector of synthesis filters with components

$$
\begin{array}{r}
{\left[\mathbf{r}\left(z_{1}, z_{2}\right)\right]_{m+M n}=z_{1}^{-(M-1-m)} z_{2}^{-(N-1-n)},} \\
0 \leq m \leq M-1, \\
0 \leq n \leq N-1 .
\end{array}
$$

If we now insert the $M N \times M N$ matrices $\mathbf{A}$ and $\mathbf{B}$ in a manner analogous to Fig. 13, then the new set of analysis and synthesis filters can be expressed in terms of $\mathbf{e}\left(z_{1}\right.$, $\left.z_{2}\right), \mathbf{r}\left(z_{1}, z_{2}\right), \mathbf{A}$ and $\mathbf{B}$. Defining the $M N \times 1$ vectors $\mathbf{h}\left(z_{1}, z_{2}\right)$ and $\mathbf{f}\left(z_{1}, z_{2}\right)$ of analysis and synthesis filters as

$$
\begin{aligned}
{\left[\mathbf{h}\left(z_{1}, z_{2}\right)\right]_{m+M n}=} & H_{m n}\left(z_{1}, z_{2}\right), \\
& {\left[\mathbf{f}\left(z_{1}, z_{2}\right)\right]_{m+M n}=F_{m n}\left(z_{1}, z_{2}\right) }
\end{aligned}
$$

for $0 \leq m \leq M-1,0 \leq n \leq N-1$, we have

$$
\mathbf{h}\left(z_{1}, z_{2}\right)=\mathbf{A e}\left(z_{1}, z_{2}\right), \quad \mathbf{f}^{T}\left(z_{1}, z_{2}\right)=\mathbf{r}^{T}\left(z_{1}, z_{2}\right) \mathbf{B} .
$$

In fact, given any set of analysis and synthesis filters with degrees in $z_{1}^{-1}$ and $z_{2}^{-1}$ not exceeding $M-1$ and $N-1$, respectively, we can always represent them in the form (32) by appropriate definitions of $\mathbf{A}$ and $\mathbf{B}$. Let us define

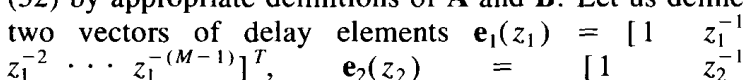




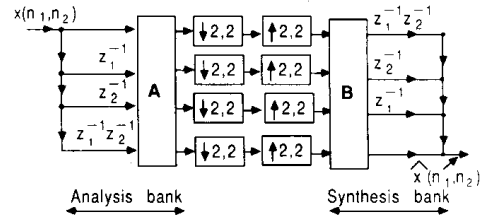

Fig. 13. The two-dimensional difference-based analysis/synthesis system redrawn. Here $M=N=2$.

$\left.z_{2}^{-2} \cdots z_{2}^{-(N-1)}\right]^{T}$. Then it is easy to see that $\mathbf{e}\left(z_{1}, z_{2}\right)$ is the Kronecker product

$$
\mathbf{e}\left(z_{1}, z_{2}\right)=\mathbf{e}_{2}\left(z_{2}\right) \otimes \mathbf{e}_{1}\left(z_{1}\right) .
$$

For example, the vector $\left[\begin{array}{llll}1 & z_{1}^{-1} & z_{2}^{-1} & z_{1}^{-1} z_{2}^{-1}\end{array}\right]^{T}$ in (28) can be written as

$$
\left[\begin{array}{c}
1 \\
z_{2}^{-1}
\end{array}\right] \otimes\left[\begin{array}{c}
1 \\
z_{1}^{-1}
\end{array}\right]
$$

We now make the observation that the set of analysis filters in (28) can also be written as a Kronecker product

$$
\begin{aligned}
{\left[\begin{array}{l}
H_{00}\left(z_{1}, z_{2}\right) \\
H_{10}\left(z_{1}, z_{2}\right) \\
H_{01}\left(z_{1}, z_{2}\right) \\
H_{11}\left(z_{1}, z_{2}\right)
\end{array}\right] } & =\left[\begin{array}{l}
1 \\
1-z_{1}^{-1} \\
1-z_{2}^{-1} \\
\left(1-z_{1}^{-1}\right)\left(1-z_{2}^{-1}\right)
\end{array}\right] \\
& =\left[\begin{array}{l}
1 \\
1-z_{2}^{-1}
\end{array}\right] \otimes\left[\begin{array}{l}
1 \\
1-z_{1}^{-1}
\end{array}\right] .
\end{aligned}
$$

In analogy with this, we define the vector of $M N$ analysis filters $\mathbf{h}\left(z_{1}, z_{2}\right)$ for the general case, as a Kronecker product

$$
\mathbf{h}\left(z_{1}, z_{2}\right)=\mathbf{h}_{2}\left(z_{2}\right) \otimes \mathbf{h}_{1}\left(z_{1}\right)
$$

where

$$
\begin{aligned}
& \mathbf{h}_{1}\left(z_{1}\right)=\left[\begin{array}{lll}
1 & \left(1-z_{1}^{-1}\right)\left(1-z_{1}^{-1}\right)^{2}
\end{array}\right. \\
& \left.\cdots\left(1-z_{1}^{-1}\right)^{M-1}\right]^{T} \\
& \mathbf{h}_{2}\left(z_{2}\right)=\left[\begin{array}{lll}
1 & \left(1-z_{2}^{-1}\right) & \left(1-z_{2}^{-1}\right)^{2}
\end{array}\right. \\
& \left.\cdots\left(1-z_{2}^{-1}\right)^{N-1}\right]^{T} \text {. }
\end{aligned}
$$

Now define $\mathbf{E}_{1}$ to be an $M \times M$ binomial matrix of the form (21), and $\mathbf{E}_{2}$ to be the corresponding $N \times N$ matrix. It is then clear that we can write $\mathbf{h}_{1}\left(z_{1}\right)=\mathbf{E}_{1} \mathbf{e}_{1}\left(z_{1}\right), \mathbf{h}_{2}\left(z_{2}\right)$ $=\mathbf{E}_{2} \mathbf{e}_{2}\left(z_{2}\right)$, so that (36) gives $\mathbf{h}\left(z_{1}, z_{2}\right)=\left\{\mathbf{E}_{2} \mathbf{e}_{2}\left(z_{2}\right)\right\}$ $\otimes\left\{\mathbf{E}_{1} \mathbf{e}_{1}\left(z_{1}\right)\right\}$. By making use of the identity (2) for Kronecker products, we obtain

$$
\mathbf{h}\left(z_{1}, z_{2}\right)=\left(\mathbf{E}_{2} \otimes \mathbf{E}_{1}\right)\left(\mathbf{e}_{2}\left(z_{2}\right) \otimes \mathbf{e}_{1}\left(z_{1}\right)\right) .
$$

In other words, we have

$$
\mathbf{h}\left(z_{1}, z_{2}\right)=\mathbf{A e}\left(z_{1}, z_{2}\right)
$$

where $\mathbf{A}=\mathbf{E}_{2} \otimes \mathbf{E}_{1}$. As an example, let $N=M=3$. The binomial matrices $\mathbf{E}_{1}$ and $\mathbf{E}_{2}$ are

$$
\mathbf{E}_{1}=\mathbf{E}_{2}=\left[\begin{array}{rrr}
1 & 0 & 0 \\
1 & -1 & 0 \\
1 & -2 & 1
\end{array}\right]
$$

so that $\mathbf{A}=\mathbf{E}_{2} \otimes \mathbf{E}_{1}$ becomes

$$
\mathbf{A}=\left[\begin{array}{rrrrrrrrr}
1 & 0 & 0 & 0 & 0 & 0 & 0 & 0 & 0 \\
1 & -1 & 0 & 0 & 0 & 0 & 0 & 0 & 0 \\
1 & -2 & 1 & 0 & 0 & 0 & 0 & 0 & 0 \\
1 & 0 & 0 & -1 & 0 & 0 & 0 & 0 & 0 \\
1 & -1 & 0 & -1 & 1 & 0 & 0 & 0 & 0 \\
1 & -2 & 1 & -1 & 2 & -1 & 0 & 0 & 0 \\
1 & 0 & 0 & -2 & 0 & 0 & 1 & 0 & 0 \\
1 & -1 & 0 & -2 & 2 & 0 & 1 & -1 & 0 \\
1 & -2 & 1 & -2 & 4 & -2 & 1 & -2 & 1
\end{array}\right] .
$$

The vector of analysis filters $\mathbf{h}\left(z_{1}, z_{2}\right)$ in this case becomes (with $g(x) \triangleq 1-x^{-1}$ )

$$
\begin{aligned}
& {\left[\begin{array}{llllll}
1 & g\left(z_{1}\right) & g^{2}\left(z_{1}\right) & g\left(z_{2}\right) & g\left(z_{2}\right) g\left(z_{1}\right) & g\left(z_{2}\right) g^{2}\left(z_{1}\right)
\end{array}\right.} \\
& \left.g^{2}\left(z_{2}\right) g^{2}\left(z_{2}\right) \quad g\left(z_{1}\right) \quad g^{2}\left(z_{2}\right) g^{2}\left(z_{1}\right)\right] .
\end{aligned}
$$

Synthesis Filters for Perfect Recovery from Undersampled Differences: Having identified the general form of $\mathbf{A}$, it remains to find $\mathbf{B}$ so that the synthesis filters $F_{m n}\left(z_{1}\right.$, $z_{2}$ ), which give rise to perfect reconstruction, can be designed. This is facilitated by use of the following lemma.

Lemma 3.3: Let $\mathbf{A}=\mathbf{E}_{2} \otimes \mathbf{E}_{1}$ where $\mathbf{E}_{2}$ and $\mathbf{E}_{1}$ are square binomial matrices of the form (21) (not necessarily of the same dimension). Then $\mathbf{A}^{-1}=\mathbf{A}$.

Indeed, a special case of this lemma for $M=N=2$ has been verified earlier in this section. The proof of the lemma follows by noting that, according to the identity (2) for Kronecker products, we have $\mathbf{A}^{2}=\left\{\mathbf{E}_{2} \otimes \mathbf{E}_{1}\right\}\left\{\mathbf{E}_{2}\right.$ $\left.\otimes \mathbf{E}_{1}\right\}=\mathbf{E}_{2}^{2} \otimes \mathbf{E}_{1}^{2}$. According to Lemma 3.1, we have $\mathbf{E}_{1}^{2}=\mathbf{I}_{M}$ and $\mathbf{E}_{2}^{2}=\mathbf{I}_{N}$ so that this reduces to $\mathbf{A}^{2}=\mathbf{I}_{M N}$ establishing the lemma.

The above results can now be state as a sampling theorem, which is the 2-D extension of the difference-sampling theorem.

Theorem 3.2: Let $x\left(n_{1}, n_{2}\right)$ be a 2-D sequence. Define the difference sequences $x_{m n}\left(n_{1}, n_{2}\right)$ according to

$$
\begin{gathered}
X_{m, n}\left(z_{1}, z_{2}\right)=\left(1-z_{1}^{-1}\right)^{m}\left(1-z_{2}^{-1}\right)^{n} X\left(z_{1}, z_{2}\right), \\
0 \leq m \leq M-1, \\
0 \leq n \leq N-1 .
\end{gathered}
$$

Let each of the $M N$ sequences $x_{m n}\left(n_{1}, n_{2}\right)$ be decimated by factors $M$ and $N$ in the horizontal and vertical directions, respectively. Then the original sequence $x\left(n_{1}, n_{2}\right)$ can be reconstructed exactly from these decimated sequences by FIR filtering operations alone. The vector of $M N$ FIR synthesis filters is given by $\mathbf{f}\left(z_{1}, z_{2}\right)=\mathbf{r}^{T}\left(z_{1}\right.$, $\left.z_{2}\right) \mathbf{A}$ with $\mathbf{A}=\mathbf{E}_{2} \otimes \mathbf{E}_{1}$ and $\mathbf{r}\left(z_{1}, z_{2}\right)$ as in (30). 


\section{Nonuniform SAmpling Theorems for Sequences}

Consider a sequence $x(n)$ with transform as shown in Fig. 2(a). As mentioned in Section I, it is possible to reduce the data rate by the factor $3 / 2$ without losing information about $x(n)$. Three methods were mentioned in Section I to accomplish this goal. The third method, which was the least conventional (and the least complicated as far as the transmitter is concerned) will now be elaborated.

The general problem we wish to consider here is this: let $x(n)$ be a sequence band-limited to $|\omega|<(L / M) \pi$, where $L$ and $M$ are integers with $L \leq M$. Imagine that the time axis is divided into intervals of length $M$. We wish to retain an arbitrary subset of $L$ samples (out of $M$ ) in each of these intervals, and discard the rest. (This subset, although arbitrary, is fixed for all intervals.) From the resulting sequence, how does the receiver recover the original sequence $x(n)$ ? In view of the "folk theorem," we expect this to be possible, and indeed, the key ingredients for obtaining such reconstruction can be inferred from the results of [5] (bunched sampling of continuous time signals). In this section we give an independent, detailed presentation directly in terms of the sequences involved, by using a polyphase filter bank framework. Design examples based on FIR reconstruction filters at the receiver end are also included.

\section{A. The $M=3, L=2$ Case}

It is most convenient to consider the special case of $M$ $=3, L=2$ in sufficient detail, and then take up the general situation. In Fig. 1, let us assume $M=3$, and consider the following peculiar choice for the three analysis filters:

$$
H_{0}(z)=1, \quad H_{1}(z)=z^{-1}, \quad H_{2}(z)=0 .
$$

Fig. 14 shows such an analysis bank. The sequences $x_{0}(n)$ and $x_{1}(n)$ contain subsets of samples of $x(n)$ as shown separately in Fig. 15. The effect of the analysis bank is merely to retain two out of three successive samples of $x(n)$ and discard the rest. Clearly, the following samples of $x(n)$ are transmitted by the analysis bank:

$$
\begin{gathered}
\cdots x(-4) x(-3) x(-1) x(0) x(2) x(3) \\
x(5) x(6) x(8) x(9) \cdots .
\end{gathered}
$$

Assuming that $x(n)$ is band-limited as in Fig. 2, we wish to obtain the two synthesis filters $F_{k}(z), k=0,1$ such that the reconstructed sequence $\hat{x}(n)$ is equal to $x(n)$ [or at least approximates a delayed version of $x(n)]$. From (8) in Section II we know that we will have $\hat{x}(n)=x(n)$ (i.e., $T(z)=1$ ) if and only if the following equations are satisfied for all $z$ :

$$
\left[\begin{array}{ll}
1 & z^{-1} \\
1 & W^{-1} z^{-1} \\
1 & W^{-2} z^{-1}
\end{array}\right]\left[\begin{array}{l}
F_{0}(z) \\
F_{1}(z)
\end{array}\right]=\left[\begin{array}{l}
3 \\
0 \\
0
\end{array}\right]
$$

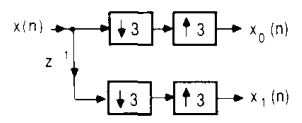

Fig. 14. The analysis bank, which retains two out of three samples from the sequence $x(n)$.

(a)

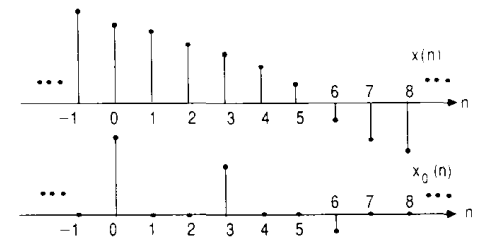

(c)

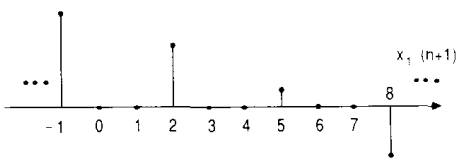

Fig. 15. Example of $x(n)$, and the subsequence $x_{0}(n)$ and $x_{1}(n)$.

We have to solve for $F_{0}(z)$ and $F_{1}(z)$ from here. Recall that the set of equations (8) came from (5) and that $m$ represents the row index of the AC matrix. Accordingly, the $m$ th equation in (8) is the necessary condition for cancelling off $X\left(z W^{m}\right)$ from $\hat{X}(z)$. For steady-state frequencies, we have $X\left(z W^{m}\right)=X\left(e^{j \omega} e^{-j 2 \pi m / 3}\right)$, since $W=$ $e^{-j 2 \pi / 3}$ for $M=3$. Fig. 16 shows sketches of $X(z W)$ and $X\left(z W^{2}\right)$

Let us assume that $F_{k}\left(e^{j \omega}\right)=0$ for $|\omega| \geq 2 \pi / 3$, so that any aliased component of $X(z)$ in this frequency range gets eliminated. It then remains only to adjust the responses of $F_{k}\left(e^{j \omega}\right)$ in the band $|\omega|<2 \pi / 3$ such that the aliased components $X(z W)$ and $X\left(z W^{2}\right)$ are cancelled. Because of the band-limited nature of $X(z)$, the quantity $X(z W)$ occupies only the region $0 \leq \omega<4 \pi / 3$ while $X\left(z W^{2}\right)=X\left(z W^{-1}\right)$ occupies the region $-4 \pi / 3<\omega$ $\leq 0$. Accordingly, we have to satisfy only two equations in each of these two frequency regions:

$$
\left[\begin{array}{ll}
1 & 1 \\
1 & W^{-1}
\end{array}\right]\left[\begin{array}{l}
F_{0}\left(e^{j \omega}\right) \\
e^{-j \omega} F_{1}\left(e^{j \omega}\right)
\end{array}\right]=\left[\begin{array}{l}
3 \\
0
\end{array}\right], \quad 0 \leq \omega<2 \pi / 3
$$

and

$$
\left[\begin{array}{ll}
1 & 1 \\
1 & W
\end{array}\right]\left[\begin{array}{l}
F_{0}\left(e^{j \omega}\right) \\
e^{-j \omega} F_{1}\left(e^{j \omega}\right)
\end{array}\right]=\left[\begin{array}{l}
3 \\
0
\end{array}\right], \quad-2 \pi / 3<\omega \leq 0
$$

Solving these, and multiplying the solutions with $e^{-j \omega}$ to avoid noncausality, we get

$$
F_{0}\left(e^{j \omega}\right)= \begin{cases}e^{-j \omega}(1-c+j s), & 0 \leq \omega<2 \pi / 3 \\ e^{-j \omega}(1-c-j s), & -2 \pi / 3<\omega \leq 0 \\ 0, & \text { otherwise }\end{cases}
$$



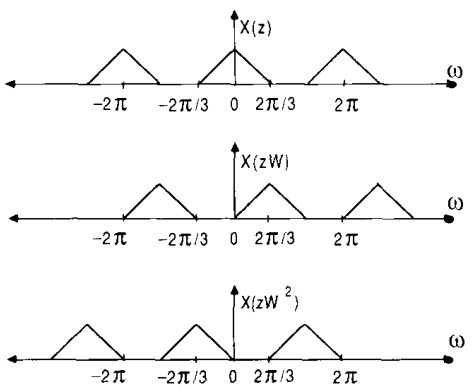

Fig. 16. The sketches of $X(z)$ and the two alias components, for $M=3$.

and

$$
F_{1}\left(e^{j \omega}\right)= \begin{cases}1-c-j s, & 0 \leq \omega<2 \pi / 3 \\ 1-c+j s, & -2 \pi / 3<\omega \leq 0 \\ 0, & \text { otherwise, }\end{cases}
$$

where we use the notations $c=\cos (2 \pi / 3)$ and $s=-\sin$ $(2 \pi / 3)$, so that $W=e^{-j 2 \pi / 3}=c+j s$. Let $G_{L}(z)$ represent an ideal low-pass filter with $G_{L}\left(e^{j \omega}\right)=1$ for $|\omega|$ $<2 \pi / 3$ and $G_{L}\left(e^{j \omega}\right)=0$ for $|\omega| \geq 2 \pi / 3$. Let $G_{H}(z)$ represent an ideal Hilbert transformer, i.e., $G_{H}\left(e^{j \omega}\right)=j$ sgn $[\omega]$. We can then write the solutions (50) and (51) as

$$
\begin{aligned}
& F_{0}(z)=z^{-1}\left\{1-c+s G_{H}(z)\right\} G_{L}(z), \\
& F_{1}(z)=\left\{1-c-s G_{H}(z)\right\} G_{L}(z) .
\end{aligned}
$$

In summary, given the sequences $x_{0}(n)$ and $x_{1}(n)$, if we pass them through the ideal filters $F_{0}(z)$ and $F_{1}(z)$, respectively, and add the results, we get back the delayed sequence $x(n-1)$ !

In practice, of course, we have to approximate the ideal responses of $G_{H}(z)$ and $G_{L}(z)$. This can be done most conveniently with linear-phase FIR filters [32], so as to avoid phase distortion completely. The low-pass filter should have a symmetric impulse response, whereas the Hilbert transformer should have an antisymmetric impulse response [32]. The specifications for the approximations are typically as shown in Fig. 18, with the bandedges located according to reconstruction accuracy desired. Let $N_{L}$ and $N_{H}$ denote the lengths of the FIR filters used for $G_{L}(z)$ and $G_{H}(z)$. Fig. 17 shows a structure for the complete analysis/synthesis system, where $K=$ $\left(N_{H}-1\right) / 2$.

The Hilbert transformer in practice has zero gain for $\omega$ $=0$, so that perfect reconstruction of $X\left(e^{j \omega}\right)$ is possibly only at frequencies excluding the neighborhood of $\omega=0$. With the specifications of Fig. 18, the region $-\theta_{p} \leq \omega$ $\leq \theta_{p}$ cannot be reconstructed. However, by making $\theta_{p}$ smaller, we can reduce this nonreconstructible neighborhood to any desired extent. Since the response of the Hilbert transformer for $|\omega|>\omega_{s}$ is immaterial (because the low-pass filter cuts it off anyway), it can be chosen to

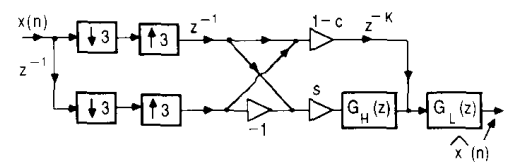

Fig. 17. The complete analysis/synthesis system for the $L=2, M=3$ example.

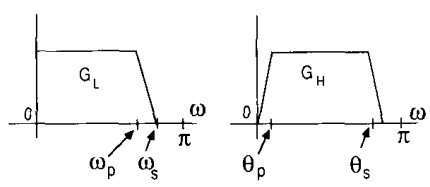

(a)

(b)

Fig. 18. Specifications of band edges for (a) the low-pass filter and (b) the Hilbert transformer, to be used with the synthesis bank.

minimize design complexity. For example, if we choose $\theta_{s}=\pi-\theta_{p}$ and design $\left|G_{H}\left(e^{j \omega}\right)\right|$ to be symmetric with respect to $\omega=\pi / 2$, then approximately 50 percent of its impulse response coefficients are equal to zero (provided $N_{H}$ is odd [32]).

Design Example 4.1: For an example of this kind of reconstruction, consider a finite-length real sequence $x(n)$ of length 71 . Due to finiteness of the length, $x(n)$ cannot be band-limited, but we can make it approximately so; the magnitude of $X\left(e^{j \omega}\right)$ is shown in Fig. 19(a), showing that $x(n)$ is essentially band-limited to the region $|\omega|<2 \pi / 3$ (the normalized frequency in the plot is defined to be $f=$ $\omega / 2 \pi$, which equals 0.5 at $\omega=\pi)$. The sequences $x_{0}(n)$ and $x_{1}(n)$ defined in Fig. 14 have transforms as shown in Fig. 19(b), and contain in-band aliasing terms (region $|\omega|$ $<2 \pi / 3$ ) and out-of-band aliasing terms (region $|\omega| \geq$ $2 \pi / 3)$. In order to reconstruct $x(n)$ using the scheme of Fig. 17, a linear phase low-pass filter $G_{L}(z)$ with parameters $\omega_{p}=0.58 \pi, \omega_{s}=0.70 \pi$, and $N_{L}=73$, which has a stopband attenuation exceeding $55 \mathrm{~dB}$, was used along with a linear-phase FIR Hilbert transformer $G_{H}(z)$ with $\theta_{p}=0.04 \pi, \theta_{s}=0.96 \pi$, and $N_{H}=51$. Fig. 20 shows the magnitude of the transform $\hat{X}\left(e^{j \omega}\right)$ of the reconstructed signal, which is seen to be very close to that of $X\left(e^{j \omega}\right)$. The responses of the filters used are shown in Fig. 21. The linear nature of their phase responses guarantees that $\hat{X}(z)$ is free from phase distortion. The total group delay caused by the analysis/synthesis system is equal to $1+\left(N_{L}-1+N_{H}-1\right) / 2=62$ samples. The time domain plots of $x(n)$ and $\hat{x}(n+62)$ are shown in Fig. 22, demonstrating the almost perfect nature of the reconstruction. Notice that it is necessary for the passbands of the two filters to be good, so that $\hat{X}\left(e^{j \omega}\right)$ is reconstructed accurately.

The effect of the low-pass filter in Fig. 17 is to eliminate the out-of-band aliasing terms, whereas the Hilbert transformer cancels the in-band aliasing terms. As a demonstration, Fig. 23 shows a plot of $\left\{\hat{X}\left(e^{j \omega}\right) \mid\right.$ with a poor Hilbert transformer $\left(N_{H}=11\right.$, and $\theta_{p}$ is larger $)$. The effect of residual in-band aliasing is evident here. 


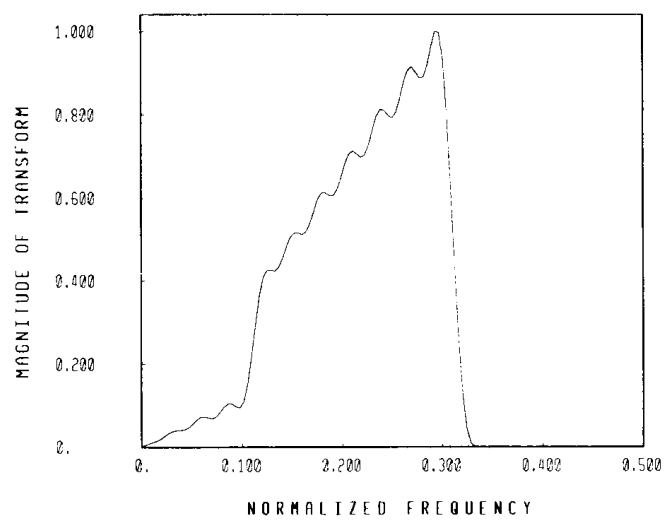

(a)

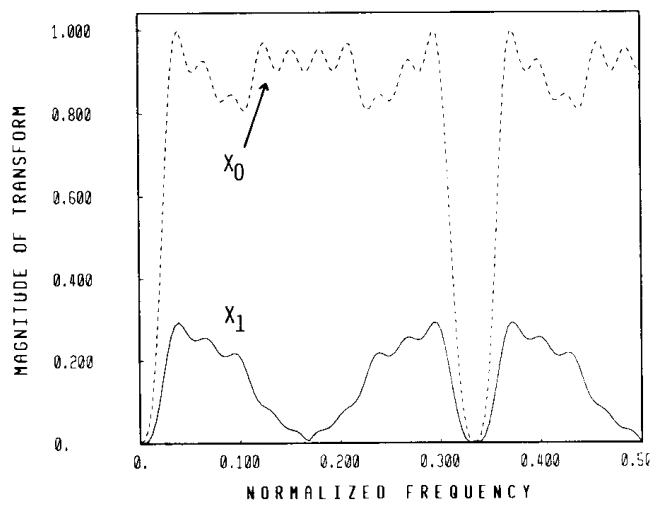

(b)

Fig. 19. (a) The quantity $\left|X\left(e^{j \omega}\right)\right|$ for Example 4.1. (b) The transforms of the decimated sequences $x_{0}(n), x_{1}(n)$.

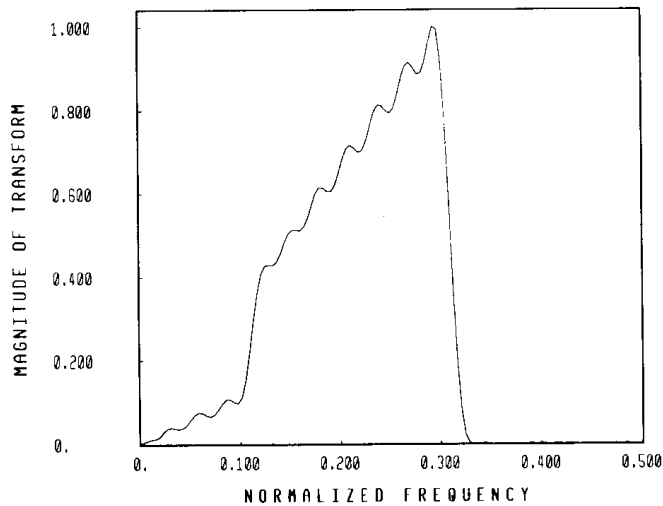

Fig. 20. The quantity $\left|\hat{X}\left(e^{j \omega}\right)\right|$ for the reconstructed sequence $\hat{x}(n)$ in Example 4.1.

Because of the use of nonideal filters $G_{L}(z)$ and $G_{H}(z)$, in practice, aliasing is not completely cancelled. Assuming that it has been reduced to the desired extent, the overall distortion transfer function $T(z)$ of $(9)$ can be written as $T(z)=\frac{1}{2}\left[H_{0}(z) F_{0}(z)+H_{1}(z) F_{1}(z)\right]$ which reduces

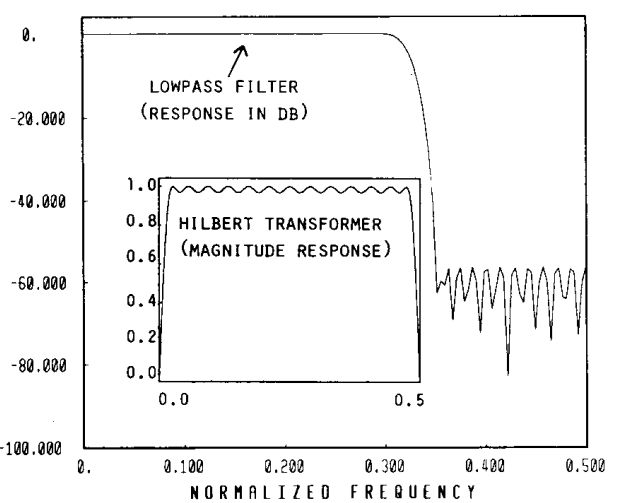

Fig. 21. Magnitude responses for the filters used in Example 4.1.

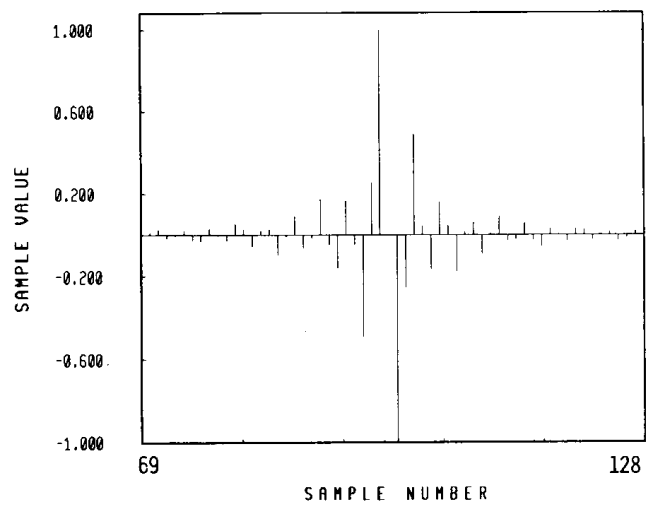

(a)

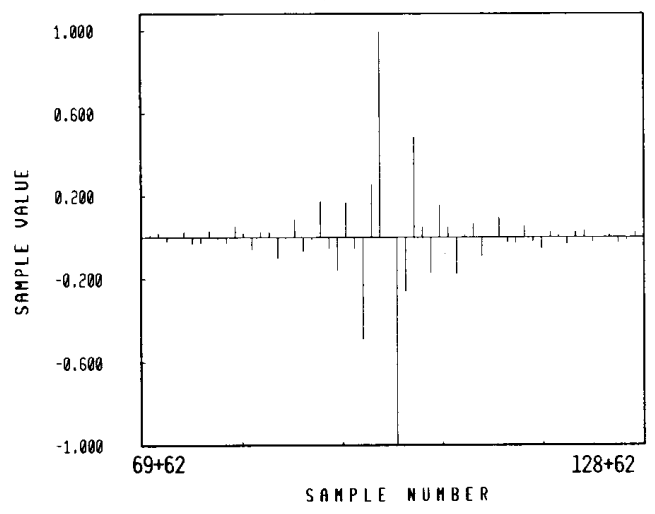

(b)

Fig. 22. (a) The sequence $x(n)$, for $69 \leq n \leq 128$. (b) The reconstructed sequence $\hat{x}(n)$ for $69+62 \leq n \leq 128+62$, for Example 4.1 .

to $(1-c) z^{-1} G_{L}(z)$. We therefore see that the imperfection of the Hilbert transformer does not affect $T(z)$ at all. It affects only the alias-component appearing around $\omega=$ 0 .

B. The General Case of Arbitrary $M, L$ with $L<M$

Assume next that $x(n)$ is band-limited to $|\omega|<L \pi / M$, where $L<M$. If $L<M / 2$, then we can decimate $x(n)$ 


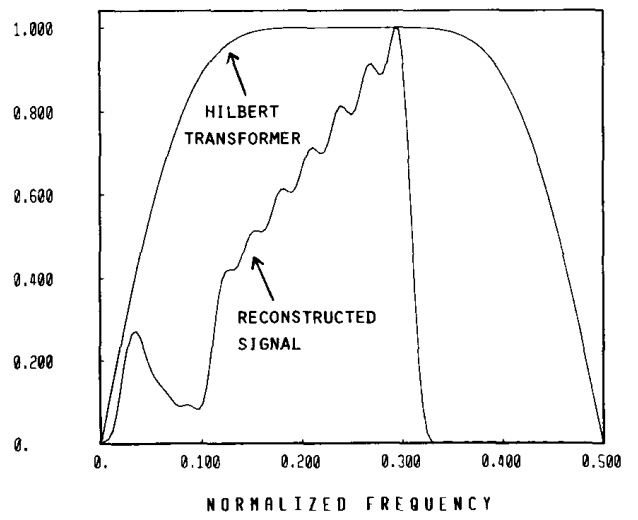

Fig. 23. The transform of the reconstructed sequence in Example 4.1, with a poor Hilbert transformer.

by an integer factor and convert the problem to one where $L>M / 2$. Accordingly, we assume $L>M / 2$ here. Referring back to the $M$-channel analysis/synthesis system, consider the choice of analysis filters

$$
H_{k}(z)= \begin{cases}z^{-n_{k}}, & 0 \leq k \leq L-1 \\ 0, & L \leq k \leq M-1 .\end{cases}
$$

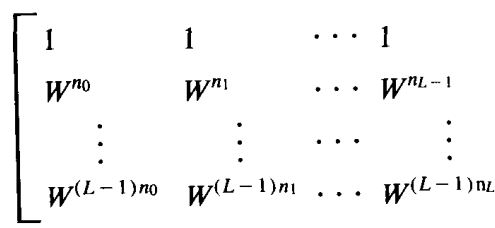

The integers $n_{k}$ are such that $0 \leq n_{0}<n_{1}<\cdots<$ $n_{L-1} \leq M-1$. Fig. 24 depicts the situation. The analysis bank therefore retains $L$ out of a set of $M$ samples; for example, in the region $-(M-1) \leq n \leq 0$, the samples numbered $-n_{L-1},-n_{L-2}, \cdots,-n_{0}$ are retained. This pattern of undersampling repeats periodically. Our task now is to find the $L$ synthesis filters $F_{n k}(z)$ such that $\hat{x}(n)$ is an accurately reconstructed version of $x(n)$. If perfect reconstruction is desired, we have to satisfy (8) with $T(z)$ equal to a constant. Thus, it is necessary and sufficient to satisfy the following equation: $\Sigma_{k=0}^{L-1}\left(z W^{m}\right)^{-n_{k}} F_{n_{k}}(z)=$ $M \delta(m)$ for $0 \leq m \leq M-1$, and for each $z$. Here $\delta(m)$ is the unit pulse function and $W=e^{-j 2 \pi / M}$. After a reordering of equations, these can be written as

$$
\sum_{k=0}^{L-1} W^{m n_{k}} z^{-n_{k}} F_{n_{k}}(z)=M \delta(m), \quad 0 \leq m \leq M-1 .
$$

Because of the band-limited nature of $X\left(e^{j \omega}\right)$, only a subset of $L$ equations in (54) are required to be satisfied for a given frequency $\omega$. Fig. 25 explains this, where the fre-

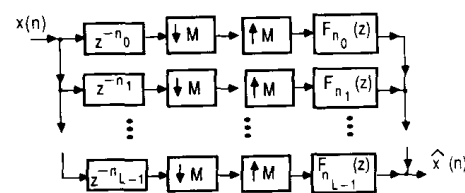

Fig. 24. The analysis-bank model for retaining $L$ out of $M$ samples, and the corresponding synthesis bank.

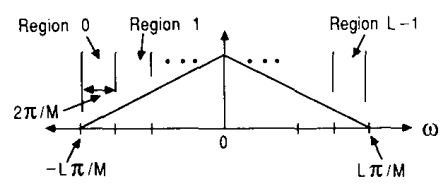

Fig. 25. The $L$ frequency regions involved.

quency range $-L \pi / M \leq \omega \leq L \pi / M$ is divided into $L$ consecutive regions of equal length $2 \pi / M$. For $0 \leq i \leq$ $L-1$, let region $i$ represent the interval $[-L \pi / M+$ $2 \pi i / M,-L \pi / M+2 \pi(i+1) / M]$. It can then be verified that the subset of $L$ equations in (54), which should be satisfied in region $i$, are the first $L-i$ equations, and the last $i$ equations. With some algebra these equations can be rearranged into

$$
]\left[\begin{array}{c}
\left(z W^{i}\right)^{-n_{0}} F_{n 0}(z) \\
\left(z W^{i}\right)^{-n_{1}} F_{n_{1}}(z) \\
\vdots \\
\left(z W^{i}\right)^{-n_{L-1} F_{n L-1}(z)}
\end{array}\right]=\left[\begin{array}{c}
0 \\
\vdots \\
0 \\
M \\
0 \\
\vdots \\
0
\end{array}\right]
$$

where the $M$ on the right-hand side occurs at the $i$ th position. The $L \times L$ matrix on the left-hand side is a Vandermonde matrix with distinct columns and is hence nonsingular. This establishes the existence of a unique set of synthesis filters (not necessarily realizable) for perfect reconstruction. Denoting the inverse of the $L \times L$ matrix in (55) by $\mathbf{U}$, we finally arrive at

$$
\mathbf{f}(z)=M \mathbf{u}_{i}, \quad \text { for region } i
$$

where $\mathbf{f}(z)$ is a column vector whose $k$ th component is $\left(z W^{i}\right)^{-n_{k}} F_{n_{k}}(z)$, and $\mathbf{u}_{i}$ is the $i$ th column of $\mathbf{U}$. Thus, the quantity $z^{-n_{k}} F_{n_{k}}(z)$ is equal to a complex constant in each of the $L$ frequency regions indicated above. Piecing together these constants gives the complete solution for the synthesis bank. In practice, however, digital filters should be used to approximate this piecewise constant response, and discontinuities occur at the junctions of the frequency regions, giving rise to reconstruction errors.

Design Example 4.2: Let $L=3, n_{0}=0, n_{1}=1$, and $n_{2}=2$. Then the matrix $\mathrm{U}$ is 


$$
\mathbf{U}=\frac{\left[\begin{array}{lll}
W^{5}-W^{4} & W^{2}-W^{4} & W^{2}-W \\
W^{2}-W^{4} & W^{4}-1 & 1-W^{2} \\
W^{2}-W & 1-W^{2} & W-1
\end{array}\right]}{W\left(W^{4}-1\right)+2 W^{2}\left(1-W^{2}\right)} .
$$

Taking for an example $M=4$, and solving for the synthesis filters, we obtain

$$
\begin{aligned}
& F_{0}\left(e^{j \omega}\right)= \begin{cases}1+j, & -\frac{3}{4} \pi<\omega \leq-\frac{\pi}{4} \\
2, & -\frac{\pi}{4} \leq \omega \leq \frac{\pi}{4} \\
1-j, & \frac{\pi}{4} \leq \omega<\frac{3}{4} \pi\end{cases} \\
& e^{-j \omega} F_{1}\left(e^{j \omega}\right)= \begin{cases}2, & -\frac{3}{4} \pi<\omega \leq-\frac{\pi}{4} \\
0, & -\frac{\pi}{4} \leq \omega \leq \frac{\pi}{4} \\
2, & \frac{\pi}{4} \leq \omega<\frac{3}{4} \pi\end{cases} \\
& e^{-2 j \omega} F_{2}\left(e^{j \omega}\right)= \begin{cases}1-j, & -\frac{3}{4} \pi<\omega \leq-\frac{\pi}{4} \\
2, & -\frac{\pi}{4} \leq \omega \leq \frac{\pi}{4} \\
1+j, & \frac{\pi}{4} \leq \omega<\frac{3}{4} \pi\end{cases}
\end{aligned}
$$

and $F_{0}\left(e^{j \omega}\right)=F_{1}\left(e^{j \omega}\right)=F_{2}\left(e^{j \omega}\right)=0$ for $|\omega|>3 \pi / 4$. These filters can be expressed in terms of ideal low-pass filters and Hilbert transformers as follows. Let $G_{L_{1}}(z)$ and $G_{L_{2}}(z)$ be ideal low-pass filters with responses as shown in Fig. 26, and let $G_{H}(z)$ be the ideal Hilbert transformer defined earlier. Then

$$
\begin{aligned}
& F_{0}(z)=\left\{1+G_{L_{2}}(z)-\left[1-G_{L_{2}}(z)\right] G_{H}(z)\right\} G_{L_{1}}(z), \\
& F_{1}(z)=2 z\left\{1-G_{L_{2}}(z)\right\} G_{L_{1}}(z), \\
& F_{2}(z)=z^{2}\left\{1+G_{L_{2}}(z)+\left[1-G_{L_{2}}(z)\right] G_{H}(z)\right\} G_{L_{1}}(z) .
\end{aligned}
$$

With causal linear-phase FIR filters of lengths $N_{L_{1}}, N_{L_{2}}$, $N_{H}$ for $G_{L_{1}}(z), G_{L_{2}}(z)$, and $G_{H}(z)$, respectively, we finally obtain a causal implementation of the system. For example, $1-G_{L_{2}}(z)$ in (61) must be replaced with $z^{-K_{2}}$ $-G_{L_{2}}(z)$ where $K_{2}=\left(N_{L_{2}}-1\right) / 2$. After working out the details, the complete synthesis bank can be expressed as

$$
\begin{aligned}
& {\left[\begin{array}{lll}
F_{0}(z) & F_{1}(z) & F_{2}(z)
\end{array}\right]} \\
& \quad=G_{L_{1}}(z)\left[\begin{array}{lllll}
1 & G_{L_{2}}(z) & G_{H}(z) & G_{L_{2}}(z) & G_{H}(z)
\end{array}\right] \mathbf{P}(z)
\end{aligned}
$$

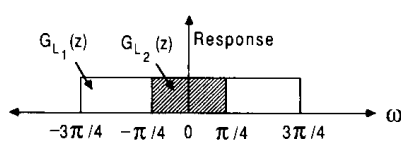

Fig. 26. The responses of the two ideal low-pass filters involved in the $M=4, L=3$ case.

where the matrix $\mathbf{P}(z)$ is given by

$$
\begin{aligned}
& \mathbf{P}(z)=\left[\begin{array}{llll}
z^{-\left(K+K_{2}\right)} & 0 & 0 & 0 \\
0 & z^{-K} & 0 & 0 \\
0 & 0 & z^{-K_{2}} & 0 \\
0 & 0 & 0 & 1
\end{array}\right]\left[\begin{array}{rrr}
1 & 1 & 1 \\
1 & -1 & 1 \\
-1 & 0 & 1 \\
1 & 0 & -1
\end{array}\right] \\
& {\left[\begin{array}{lll}
z^{-2} & 0 & 0 \\
0 & 2 z^{-1} & 0 \\
0 & 0 & 1
\end{array}\right]}
\end{aligned}
$$

with $K=\left(N_{H}-1\right) / 2$ and $K_{2}=\left(N_{L_{2}}-1\right) / 2$. The resulting synthesis bank structure is as shown in Fig. 27.

\section{Relation to Continuous-Time Sampling}

In Fig. 24, $L$ samples are retained out of every $M$ samples. Let $m_{k}, 0 \leq k \leq L-1$ denote the sample numbers for the samples retained in the region $0 \leq n \leq M-1$. Clearly, $0 \leq m_{0}<m_{1} \cdots<m_{L-1} \leq M-1$. Let $y(k)$ be the sequence obtained by undersampling $x(n)$ in this manner. Then $y(k)=x\left(n_{k_{0}}+k_{1} M\right)$, where $k_{0}=k \bmod$ $L$ and $k=k_{0}+k_{1} L$.

Suppose that $x(n)$ has been obtained by uniform sampling of a signal $x_{a}(t)$, i.e., $x(n)=x_{a}\left(n T_{s}\right)$ where $T_{s}$ is the sampling period. Then the set of samples $y(k)$ can be regarded as nonuniformly spaced samples of $x_{a}(t)$. More specifically, $y(k)=x_{a}\left(t_{k}\right)$, where $t_{k}=\left[n_{k_{0}}+k_{1} M\right] T_{s}$. In Section IV-B, we saw that $x(n)$ can be recovered from $y(k)$ (as long as the bandwidth requirements are satisfied). In other words, by knowing the nonuniformly spaced samples of $x_{a}(t)$, the corresponding uniformly spaced samples $x_{a}\left(n T_{s}\right)$ can be recovered. Since $x_{a}\left(n T_{s}\right)$ represents an oversampled version of $x_{a}(t)$ (for $L<M$ ), we can recover $x_{a}(t)$ from $x_{a}\left(n T_{s}\right)$ in the traditional way (low-pass filtering). This proves the "nonuniform sampling theorem" for continuous time signals, under the assumptions that 1) sampling times are integer multiples of a real number $T_{s}$, and 2 ) the sampling pattern repeats periodically with period $M T_{s}$. The first assumption is equivalent to saying that the ratio of any two sampling times $t_{k}$ and $t_{n}$ is rational. This is not a serious loss of generality in practice. The second assumption may not always be valid. However, by letting the integer $M$ in Fig. 24 to get arbirarily large, one can make an asymptotic argument in this case, to understand more general versions of the " folk theorem." 


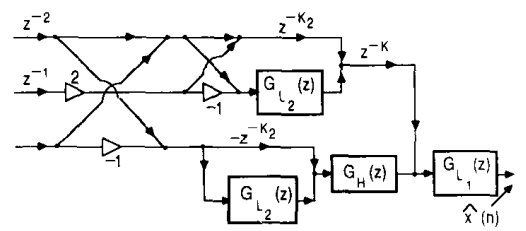

Fig. 27. A causal implementation of the complete linear-phase synthesis bank for the $M=4, L=3$ example.

\section{Concluding Remarks}

The main emphasis of this paper has been to apply the structural framework of Fig. 1 in order to enhance the understanding of several sampling theorems for sequences. Unlike in the continuous-time world, it is possible to reconstruct a sequence exactly from generalized subsamples, and such reconstruction can sometimes be done with no multiplication operations. Indeed, there exists a whole family of perfect reconstruction systems with FIR filters for $H_{k}(z)$ and $F_{k}(z)$, all of which in principle lead to a family of sampling theorems. The polyphase formulations (Figs. 9 and 10) are found to be particularly useful in obtaining most of these theorems in a simple manner. These same formulations have been used in the past for the design of the analysis filters with optimal stopband attenuation, under the constraint of perfect reconstructibility of $x(n)$.

\section{REFERENCES}

[1] C. E. Shannon, "Communications in the presence of noise," Proc. IRE, vol. 37, pp. 10-21, Jan. 1949.

[2] J. M. Whittaker, "The Fourier theory of the cardinal functions,' Proc. Math. Soc. Edinburgh, vol. 1, pp. 169-176, 1929.

[3] H. Nyquist, "Certain topics in telegraph transmission theory," AIEE Trans., vol. 47, pp. 617-644, 1928.

[4] A. J. Jerri, "The Shannon sampling theorem-Its various extensions and applications: A tutorial review,"' Proc. IEEE, pp. 1565-1596, Nov. 1977.

[5] A. Papoulis, "Generalized sampling expansion," IEEE Trans. Circuits Syst., vol. CAS-24, pp. 652-654, Nov. 1977.

[6] J. L. Brown, "Multichannel sampling of lowpass signals," IEEE Trans. Circuits Syst., vol. CAS-28, pp. 101-106, Feb. 1981.

[7] H. S. Black, Modulation Theory. New York: Van Nostrand, 1953.

[8] P. P. Vaidyanathan and V. C. Liu, "Sampling theorems in the context of polyphase digital filter banks," in Proc. 21st Annu. Asilomar Conf. Signals, Syst., Comput., Pacific Grove, CA, Nov. 1987 (to appear).

[9] R. W. Schafer and L. R. Rabiner, "Design of digital filter banks for speech analysis," Bell Syst. Tech. J., vol. 50, pp. 3097-3115, Dec. 1971.

[10] R. E. Crochiere and L. R. Rabiner, Multirate Digital Signal Processing. Englewood Cliffs, NJ: Prentice-Hall, 1983.

[11] M. Bellanger, G. Bonnerot, and M. Coudreuse, "Digital filtering by polyphase network: Application to sample rate alteration and filter banks," IEEE Trans. Acoust., Speech, Signal Processing, vol. ASSP24, pp. 109-114, Apr. 1976

[12] P. P. Vaidyanathan, "Quadrature mirror filter banks, $M$-band extensions and perfect-reconstruction techniques," IEEE ASSP Mag., to appear, 1987.

[13] A. Croisier, D. Esteban, and C. Galand, "Perfect channel splitting by use of interpolation/decimation/tree decomposition techniques," presented at the Int. Symp. Info., Circuits, Syst., Patras, Greece, 1976.

[14] J. W. Woods and S. D. O'Neil, "Subband coding of images," IEEE Trans. Acoust., Speech, Signal Processing, vol. ASSP-34, pp. 12781288 , Oct. 1986

[15] M. J. T. Smith and S. L. Eddins, "Subband coding of images with octave band tree structures," in Proc. IEEE Int. Conf. ASSP, Dallas, TX, Apr. 1987, pp. 1382-1385.

[16] P. P. Vaidyanathan, "Perfect reconstruction QMF banks for two-dimensional applications," IEEE Trans. Circuits Syst, , vol. CAS-34, pp. 976-978, Aug. 1987.

[17] M. R. Portnoff, "Time-frequency representation of digital signals and systems based on short-time Fourier analysis," IEEE Trans. Acoust., Speech, Signal Processing, vol. ASSP-28, pp. 55-69, Feb. 1980.

[18] D. E. Knuth, The Art of Computer Programming, Vol. 2-Seminumerical Algorithms. Reading, MA: Addison-Wesley, 1969.

[19] R. E. Blahut, Fast Algorithms for Digital Signal Processing. Reading, MA: Addison-Wesley, 1985.

[20] T. H. Ramstad, "Analysis/synthesis filter banks with critical sampling," presented at the Conf. Digital Signal Processing, Florence, Italy, Sept. 1984.

[21] M. J. T. Smith and T. P. Barnwell, III, "A unifying framework for analysis/synthesis systems based on maximally decimated filter banks," in Proc. IEEE Int. Conf. ASSP, Tampa, FL, Mar. 1985, pp. 521-524.

[22] M. Vetterii, "A theory of multirate filter banks," IEEE Trans. Acoust., Speech, Signal Processing, vol. ASSP-35, pp. 356-372. Mar. 1987.

[23] K. Swaminathan and P. P. Vaidyanathan, "Theory and design of uniform DFT, parallel, quadrature mirror filter banks," IEEE Trans. Circuits Syst., vol. CAS-33, pp. 1170-1191, Dec. 1986.

[24] P. P. Vaidyanathan, "Theory and design of $M$-channel maximally decimated quadrature mirror filters with arbitrary $M$, having perfect reconstruction property," IEEE Trans. Acoust., Speech, Signal Processing, vol. ASSP-35, pp. 476-492, Apr. 1987.

[25] M. Vetterli, "Splitting a signal into sub-sampled channels allowing perfect reconstruction," in Proc. IASTED Conf. Appl. Signal Processing Digital Filter., Paris, France, June 1985.

[26] M. J. T. Smith and T. P. Barnwell, III, "Exact reconstruction techniques for tree-structured subband coders," IEEE Trans. Acoust., Speech, Signal Processing, vol. ASSP-34, pp. 434-441, June 1986.

[27] P. P. Vaidyanathan, Z. Doganata, and T. Q. Nguyen, "More results on the perfect reconstruction problem in $M$-band parallel QMF bank," in Proc. IEEE Int. Symp. Circuits Syst., Philadelphia, PA, May 1987, pp. $847-850$.

[28] A. M. Davis, "Almost periodic extension of band-limited functions and its applications to nonuniform sampling," IEEE Trans. Circuits Syst., vol. CAS-33, pp. 933-936, Oct. 1986.

[29] G. Wackersreuther, "On two-dimensional polyphase filter banks," IEEE Trans. Acoust., Speech, Signal Processing, vol. ASSP-34, pp. 192-199, Feb. 1986.

[30] M. Vetterli, "Multi-dimensional sub-band coding: Some theory and algorithms," Signal Processing, vol. 6, pp. 97-112, Apr. 1984.

[31] N. S. Jayant and P. Noll, Digital Coding of Waveforms. Englewood Cliffs, NJ: Prentice-Hall, 1984.

[32] L. R. Rabiner and B. Gold, Theory and Application of Digital Signal Processing. Englewood Cliffs, NJ: Prentice-Hall, 1975.

[33] V. K. Jain and R. E. Crochiere, "Quadrature mirror filter design in the time domain," IEEE Trans. Acoust., Speech, Signal Processing, vol. ASSP-32, pp. 353-361, Apr. 1984. 
[34] C. R. Galand and H. J. Nussbaumer, "New quadrature mirror filter structures," IEEE Trans. Acoust., Speech, Signal Processing, vol. ASSP-32, pp. 522-531, June 1984.

[35] P. L. Chu, "Quadrature mirror filter design for an arbitrary number of equal bandwidth channels," IEEE Trans. Acoust., Speech, Signal Processing, vol. ASSP-33, pp. 203-218, Feb. 1985.

[36] J. H. Rothweiler, "Polyphase quadrature filters, A new subband coding technique," in Proc. IEEE Int. Conf. ASSP, Boston, MA, Apr. 1983, pp. 1980-1983.

[37] B. Kedem, "Spectral analysis and discrimination by zero-crossings," Proc. IEEE, vol. 74, pp. 1477-1493, Nov. 1986.

[38] Y. C. Jenq, "Digital spectra of nonuniformly sampled signals: Theories and applications," IEEE Trans. Instrum. Meas., vol. 37, pp. 245-251, June 1988. "Digital spectra of nonuniformly sampled signals-Digital lookup tunable sinusoidal oscillators,' IEEE Trans. Instrum. Meas., vol. 37, pp. 358-362, Sept. 1988.

[39] A. Papoulis, Signal Analysis. New York: McGraw-Hill, 1977.

[40] A. V. Oppenheim, A. S. Willsky, and I. T. Young, Signals and Systems. Englewood Cliffs, NJ: Prentice-Hall, 1983.
P. P. Vaidyanathan (S'80-M'83-SM'88), for a photograph and biography, see p. 94 of the January 1988 issue of this TRANSACTIONS.

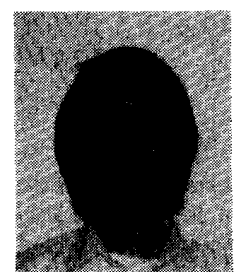

Vincent C. Liu (S'85) was born in Hong Kong on November 15, 1961. He received the B.E. degree in electrical engineering from the State University of New York at Stony Brook in 1984, and the M.S. degree from the California Institute of Technology in 1985.

$\mathrm{He}$ is currently a $\mathrm{Ph} . \mathrm{D}$. candidate at the California Institute of Technology. His main research interests are in the areas of digital signal processing, nonlinear effects in digital filters, and twodimensional discrete-time systems.

Mr. Liu is the recipient of a Schlumberger Fellowship, and is a member of Tau Beta Pi. 